OPEN ACCESS

Edited by:

Prabhat Purbey,

University of California, Los Angeles,

United States

Reviewed by:

Wei Seong Toh,

National University of Singapore,

Singapore

Masoud Mozafari,

University of Toronto, Canada

*Correspondence:

Dong-Hwee Kim

donghweekim@korea.ac.kr

Specialty section:

This article was submitted to

Cellular Biochemistry,

a section of the journal

Frontiers in Cell and Developmental

Biology

Received: 26 July 2021 Accepted: 10 November 2021 Published: 03 December 2021

Citation:

Shafiq M, Ali O,

Han S-B and

Kim D-H (2021) Mechanobiological

Strategies to Enhance Stem Cell

Functionality for Regenerative

Medicine and Tissue Engineering.

Front. Cell Dev. Biol. 9:747398.

doi: 10.3389/fcell.2021.747398

\section{Mechanobiological Strategies to Enhance Stem Cell Functionality for Regenerative Medicine and Tissue Engineering}

\author{
Muhammad Shafiq ${ }^{1}$, Onaza Ali ${ }^{2}$, Seong-Beom $\mathrm{Han}^{3}$ and Dong-Hwee Kim ${ }^{3,4 *}$ \\ ${ }^{1}$ Department of Biotechnology, Faculty of Life Sciences, University of Central Punjab, Lahore, Pakistan, ${ }^{2}$ School of Chemistry and \\ Chemical Engineering, Tiangong University, Tianjin, China, ${ }^{3} \mathrm{KU}-\mathrm{KIST}$ Graduate School of Converging Science and Technology, \\ Korea University, Seoul, South Korea, ${ }^{4}$ Department of Integrative Energy Engineering, College of Engineering, Korea University, \\ Seoul, South Korea
}

Stem cells have been extensively used in regenerative medicine and tissue engineering; however, they often lose their functionality because of the inflammatory microenvironment. This leads to their poor survival, retention, and engraftment at transplantation sites. Considering the rapid loss of transplanted cells due to poor cell-cell and cellextracellular matrix (ECM) interactions during transplantation, it has been reasoned that stem cells mainly mediate reparative responses via paracrine mechanisms, including the secretion of extracellular vesicles (EVs). Ameliorating poor cell-cell and cell-ECM interactions may obviate the limitations associated with the poor retention and engraftment of transplanted cells and enable them to mediate tissue repair through the sustained and localized presentation of secreted bioactive cues. Biomaterial-mediated strategies may be leveraged to confer stem cells enhanced immunomodulatory properties, as well as better engraftment and retention at the target site. In these approaches, biomaterials have been exploited to spatiotemporally present bioactive cues to stem cellladen platforms (e.g., aggregates, microtissues, and tissue-engineered constructs). An array of biomaterials, such as nanoparticles, hydrogels, and scaffolds, has been exploited to facilitate stem cells function at the target site. Additionally, biomaterials can be harnessed to suppress the inflammatory microenvironment to induce enhanced tissue repair. In this review, we summarize biomaterial-based platforms that impact stem cell function for better tissue repair that may have broader implications for the treatment of various diseases as well as tissue regeneration.

Keywords: biomaterials, stem cell, mechanobiology, cell fate modulation, immunosuppression, cell therapy, tissue engineering, regenerative medicine

\section{INTRODUCTION}

Stem cell therapy has garnered extensive attention and been investigated for the treatment of various pathologies. This is mainly because of the multilineage differentiation capability of stem cells, their secretion of paracrine factors, including extracellular vesicles (EVs), exosomes, ectosomes (microparticles/ macrovesicles), and their immunomodulatory properties. Consequently, stem cell therapy has been used for the regeneration of a multitude of tissues, such as infarcted myocardium, spinal cord, nerves, and skin, 
Cell Transplantation

(Intravenous, Intracardiac, Intravitreal)



In-vitro cell culture

$\downarrow$

Cell-cell contact $\downarrow$

Cell-ECM contact $\downarrow$

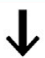

Cell appoptosis $\uparrow$

Cell servival

$\mathbf{y}$

Overall cell loss 1

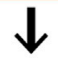

Functional tissue regeneration $\downarrow$ Scar like tissue 1
FIGURE 1 | Limitations of direct cell transplantation. Cells can be transplanted in vivo by different routes, such as intravenous, intramuscular, intra-cardiac, or intra-vitreous injection. The direct transplantation of stem cells and progenitor cells without using biomaterial platforms may lead to the poor cell-cell and cell-ECM interactions, resulting into the severe loss of the cells through apoptosis in vitro and in vivo. Moreover, the cells can experience rapid loss upon transplantation in vivo due to poor cell survival, retention, and engraftment at the injury site. Poor cell homing and immune rejection may further aggravate this problem. Overall, this can lead to severe cell loss and less functional, more scar-like tissue repair.

as well as to treat vascular injuries, ocular defects, and melanoma (Karantalis and Hare, 2015; Trounson and McDonald, 2015; Parmar et al., 2020). While implanted stem cells participate in the regenerative process by differentiating into tissue-specific cells, their poor survival and retention in vivo hamper tissue repair (Figure 1). Recently, stem cell labeling techniques using traceable nanoparticles and fluorescent dyes have been used to precisely track the survival and integration of transplanted cells in the targeted tissues (Teo et al., 2020). These factors have fueled research to elucidate the involvement of paracrine factors, including EVs in the tissue repair process.

Extensive reports have discussed the therapeutic potential of stem cell grafting into injured or defective organs. Improving the survival and retention of transplanted stem cells may help in elucidating the role of cell-mediated paracrine factors, and EVs involved in this process (Table 1) (Jha et al., 2015a; Jha et al., 2015b). Therefore, ensuring stem cell survival during transplantation and in vivo is crucial for the success of regenerative medicine and tissue engineering approaches. There is currently a good understanding of cell survival mechanisms, including cell-cell and cell-extracellular matrix (ECM) crosstalk. Studies to address the rapid loss of transplanted cells and their poor survival in vivo have used different biomaterial candidates, such as hydrogels, engineered cell sheets, instructive biomaterials, and ECM-mimicking platforms and have improved our understanding of cell loss during and after transplantation (Shafiq et al., 2016). The loss of cell-cell and cell-ECM crosstalk is believed to be the primary cause of cell loss in vitro, but cell preconditioning, licensing, and instructive biomaterials could be used to overcome this problem. Additionally, the immunosuppressive microenvironment and inflammatory response at the target site are perceived to compromise cellmediated functions, and this problem has been addressed by designing bioactive cue-tethered biomaterials (Hettiaratchi et al., 2016), including heparin-conjugated materials to sequester heparin-binding growth factors and interferon-gamma (IFNy)-tethered hydrogels for immune suppression (Figure 2). Furthermore, engineering the microenvironment at the site of injury has been pursued to improve the survival and engraftment of transplanted cells. Biomaterial-mediated delivery of cytokines and chemokines, such as transforming growth factor-beta (TGF- $\beta$ ), has been exploited to induce immune suppression in the injury microenvironment (Liu et al., 2016a).

Similarly, the use of instructive biomaterials possessing mechanical properties similar to those of native tissues and incorporating different growth factors has been proposed to enhance the survival and integration of transplanted cells. Because most of the transplanted cells are lost owing to differences in mechanical properties between the hydrogels used for cell transplantation and the loss of cell-cell and cell-ECM contacts, the use of synthetic hydrogels possessing shearthinning and self-healing characteristics has been pursued to improve the injectability of the cells into tissues in vivo in a non-invasive manner. Additionally, to mimic the cell niche-like microenvironment in vitro, ECM and ECM-derived peptide sequences and molecules capable of interacting with cellular receptors, such as hyaluronic acid (HA), have been integrated into biomaterials (Somaa et al., 2017). The microenvironment at the injury site leverages signaling cues, such as stromal cell-derived factor 1 alpha (SDF-1 $\alpha$ ) and CXC chemokine receptor 4 (CXCR4) signaling, to enhance stem cell and progenitor cell mobilization and recruitment. Instructive biomaterials possessing these cues have been shown to improve stem cell function in vitro and in vivo. However, implementing these methods requires additional strategies such as inducing vascular-like networks into the biomaterials to enhance their cellular integration via anastomosis between the transplanted cells and tissues and the host tissues or organs. These strategies have been extensively detailed elsewhere (Shafiq et al., 2016) and are thus not discussed in this review. The subsequent four sections of this review discuss some of the recent strategies employed to overcome several challenges in stem cell therapy.

\section{LOCAL PRESENTATION OF BIOACTIVE CUES TO ENHANCE STEM CELL SURVIVAL AND RETENTION \\ Engineered Biomaterials for Protein Sequestration}

Biomaterials are engineered materials that interact with host tissues and assist in their smooth functioning or induce the 
TABLE 1 | List of abbreviations.

\begin{tabular}{|c|c|c|c|}
\hline Full name & Abbreviation & Full name & Abbreviation \\
\hline Glycosaminoglycans & GAGs & Manganese oxide & $\mathrm{MnO}_{2}$ \\
\hline Bone morphogenetic protein-2 & BMP-2 & Brain-derived neurotrophic factor & BDNF \\
\hline Mesenchymal stem cells & MSCs & Glial-derived neurotrophic factor & GDNF \\
\hline CXC chemokine receptor 4 & CXCR4 & Polycaprolactone & PCL \\
\hline Insulin-like growth factor 1 & IGF-1 & Neural stem cells & NSCs \\
\hline Vascular endothelial growth factor & VEGF & Induced-pluripotent stem cells & iPSCs \\
\hline Interferon-gamma & IFN- $\gamma$ & Cardiomyocytes & $\mathrm{CMs}$ \\
\hline Hyaluronic acid & $\mathrm{HA}$ & Beta cyclodextrin & $\beta-C D$ \\
\hline Transforming growth factor-beta & TGF- $\beta$ & Mitogen-activated protein kinase & MAPK \\
\hline Monocyte chemoattractant protein 1 & MCP-1 & Hydrogen peroxide & $\mathrm{H}_{2} \mathrm{O}_{2}$ \\
\hline Interleukin-12 & IL-12 & Magnesium oxide & $\mathrm{MgO}_{2}$ \\
\hline Tumor necrosis factor-alpha & TNF- $\alpha$ & Cardiac stem cells & CSCs \\
\hline Human umbilical vein endothelial cells & HUVECs & Poly (vinyl alcohol) & PVA \\
\hline Cardiac progenitor cells & CPCs & Polyethylene glycol & PEG \\
\hline Smooth muscle cells & SMCs & Polythioketal & PTK \\
\hline Adipose-derived stem cells & ADSCs & Nanoparticles & NPs \\
\hline Bioluminescent imaging & BLI & Endothelial progenitor cells & EPCs \\
\hline Schwann cells & SCs & Extracellular domain-1 & ECD-1 \\
\hline Hematopoietic stem cells & HSCs & Poly (N-isopropylacrylamide) & PNIPAM \\
\hline Poly (L-lactide-co-glycolide) & PLGA & Adhesive protein-based immiscible condensed liquid systems & APICLS \\
\hline Epigallocatechin gallate & EGCG & Prostaglandin E2 & PGE-2 \\
\hline
\end{tabular}
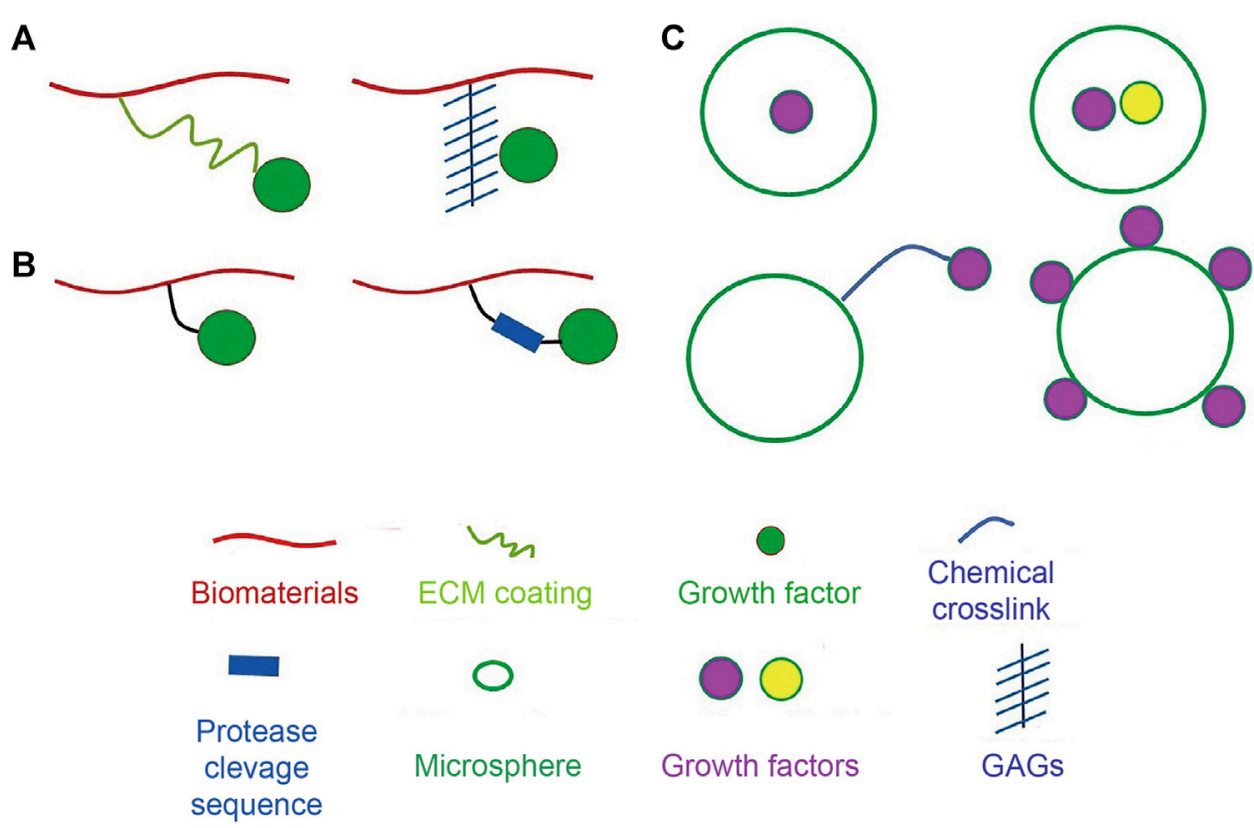

u

ECM coating

0

Microsphere

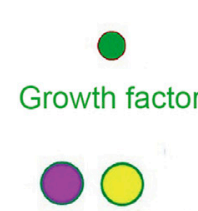

Growth factors

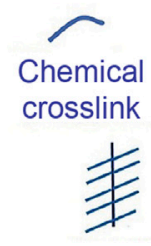

GAGs

FIGURE 2 | Strategies for attachment of bioactive molecules to biomaterials. (A) Strategies to non-covalently attach bioactive molecules to biomaterials using physical tools. Bioactive molecules can be absorbed on the surface of the biomaterial through coating ECM proteins or can be electrostatically associated with biomaterials via heparin linkage. (B) Strategies to covalently attach bioactive molecules using chemical tools. Protease-degradable amino acids sequences can be introduced into crosslinks to instruct release of signaling cues. (C) Methodologies for incorporation of bioactive molecules to microspheres. Bioactive molecules can be encapsulated, adsorbed on the surface, or chemically linked to the microspheres. Adapted with permission from (Shafiq et al., 2016). Copyrights reserved Elsevier.

regeneration of injured tissues or organs (Abdulghani and Mitchell, 2019). For instance, dental fillers and other implants, such as those used for the replacement of the hip and joints, to remediate bone and spinal fusion, or for vascular reconstruction, all fall under this category. Depending on their intended application, biomaterials can be either bio-inert, that is, biologically inert to the body or instructive, that is, leveraging biochemical cues for neo-tissue regeneration or the restoration of a lost function (Pavlovic, 2015). Bioinert materials are generally either biocompatible metals, such as titanium, tantalum, or 
zirconia, or non-degradable polymers, including polymethyl methacrylate (PMMA) and expanded polytetrafluoroethylene (ePTFE). They do not degrade in vivo, and may need to be removed at some point after implantation. In contrast, the latter class includes biomaterials that can interact with the host cells and leverage biochemical signals for neo-tissue regeneration or restoration of a lost function. These materials include biocompatible and biodegradable metals such as magnesium, as well as polymeric materials such as poly (L-lactide) (PLLA), polycaprolactone (PCL), polyglycolic acid (PGA), poly (L-lactideco-glycolic acid) (PLGA), and poly (L-lactide-co-e-caprolactone) (PLCL). Because tissues and the extracellular matrix (ECM) dynamically interact with the in vivo microenvironment, dynamic biomaterials that can reciprocally interact with the in vivo microenvironment, while perceiving and leveraging biochemical signals, including sensing force or even remodeling, have recently been proposed (Zhang et al., 2020).

Biomaterial scaffolds should be biocompatible with the host tissues. A multitude of features, including tissue- or organspecific biophysical properties and bioactive cues, are part of these biomaterials to help them create a conducive environment for tissue regeneration (Zhao et al., 2019). Another crucial aspect is the biodegradability of the biomaterials. The degradation products should not be toxic to avoid inflammatory responses. Biomaterials encompass metals, ceramics, and polymers, and the latter can be either natural or synthetic. Natural polymers, including ECM-like proteins such as fibronectin, laminin, collagen, polysaccharides, and glycosaminoglycans are widely used as biological vehicles and scaffolds because of their biocompatibility and biodegradability. Despite these interesting features, the use of natural polymers is mainly impeded by their poor mechanical properties. An advantage of using synthetic polymers, such as PCL, PLCL, and PLGA is that good control can be exercised over their synthesis and modification (Cameron and Shaver, 2011). Because synthetic polymers lack bioactivity and cell recognition factors, different types of bioactive factors, including growth factors and biologics, can be incorporated into them (Rafique et al., 2021). The second type of biomaterials, designated as natural biomaterials, is derived from natural sources such as polysaccharides and proteins. These biomaterials exhibit high biocompatibility and biodegradability, as well as unique structures that allow the mimicking of the ECM microenvironment. Polysaccharidebased biomaterials include chitosan, alginate, hyaluronic acid, and fibrin, among which chitosan is one of the most widely used cationic polymers used to deliver anionic substances, including growth factors, cytokines, genes, and glycosaminoglycans. Among protein-based biomaterials, including collagen, fibrin, gelatin, and silk, collagen is the most widely used natural biomaterial. Its advantages include simple processing, minimal induction of inflammatory reactions, and approved use by the U.S. Food and Drug Administration (FDA) (7).

Biomaterial-based systems have been exploited to facilitate in vitro and in vivo tissue repair. Controlled delivery systems for proteins have been used in the instruction of cellular processes, including motility, proliferation, and differentiation (Sneider et al., 2019; Park et al., 2020b; Lee et al., 2021). However, the inherent instability of proteins and their cost are the major limitation for this approach. Under in vivo conditions, cellsecreted proteins are sequestered by glycosaminoglycans (GAGs), such as heparin, heparan sulfate, and chondroitin sulfate, which precisely modulate the cellular processes mediated by sequestered proteins (Freudenberg et al., 2016; Hettiaratchi et al., 2016). Positively charged amino acid residues and negatively charged GAGs interact electrostatically, resulting in the sequestration of morphogens and chemokines in vivo. This has been mimicked in vitro by designing multiple biomaterial platforms capable of sequestering cell-secreted proteins to modulate cell differentiation and metabolism and impact the grafting of transplanted cells for functional tissue repair (Figure 2 and Table 2). Additionally, the preferential sequestration of cytokines and chemokines can be exploited to modulate inflammation and tissue repair processes (Martino et al., 2011; Martino et al., 2014; Lohmann et al., 2017).

Rinker et al. leveraged protein-sequestering heparin sulfate microparticles and successfully sequestrated cell-secreted proteins, which resulted in a reduction in the chondrogenic differentiation of cells in vitro (Rinker et al., 2018). Similarly, matrix metalloproteinase (MMP)-degradable hydrogels have been designed to sequester recombinant tissue inhibitors of MMPs (such as rTIMP3) via electrostatic interactions. The targeted delivery of rTIMP3-sequestering hydrogels attenuated adverse left ventricular remodeling in a porcine model of myocardial infarction (Purcell et al., 2014).

Using polyelectrolyte films, Liu et al. presented SDF-1 $\alpha$ to the ventral side of breast cancer cells, which acted synergistically with CD44 and CXCR4 and impacted cell spreading, as evidenced by the improved formation of lamellipodia and filopodia (Liu et al., 2017b). In contrast, soluble SDF-1a did not affect cellular spreading and migration. Similarly, bone morphogenetic protein-2 (BMP-2) has been locally presented using a soft polymeric film to promote osteogenesis and bone formation by mesenchymal stem cells (MSCs) in vitro (Crouzier et al., 2011). It is worth noting that in vivo growth factors are present in bound forms in the ECM and are presented to the cells in a spatiotemporal manner (Hettiaratchi et al., 2016). While substantial data exist regarding the modification of polymer scaffolds by ECM proteins and ligands, such as arginineglycine-aspartic acid (RGD), few studies have discussed the presentation of matrix-bound proteins. Griffith et al. demonstrated that matrix-bound epidermal growth factor (EGF) significantly affects cellular processes (Fan et al., 2007). Recently, matrix-bound growth factors, such as vascular endothelial growth factor (VEGF) and insulin-like growth factor 1 (IGF-1), have been shown to enhance stem cell function and tissue regeneration (Davis et al., 2006; Chen et al., 2010; Shafiq et al., 2018; Wang et al., 2020).

\section{Investigating Cellular Functions Using Protein-Sequestering Biomaterials}

Tethering of cell-instructive biomolecules on biomaterials may assist in the investigating their cellular functions. The immobilization of biomolecules may not only provide the 
TABLE 2 | Enhancing stem cell function using matrix-anchored bioactive cues.

\begin{tabular}{|c|c|c|c|c|}
\hline Biomaterial category & Bioactive cue & Cell/tissue type & Observed effect & References \\
\hline Heparin-PEG & IGFBP-3, IGFBP-5 & ATDC5 & Chondrogenic differentiation $\downarrow$ & Rinker et al. (2018) \\
\hline $\begin{array}{l}\text { Polyelectrolyte multilayered film } \\
\text { PLL/HA }\end{array}$ & SDF-1a & Breast cancer cells & Cell spreading $\downarrow$ & Picart et al., 2017 \\
\hline Soft polymer film & BMP-2 & MSCs & $\begin{array}{l}\text { Osteogenic differentiation } \uparrow \\
\text { Bone formation } \uparrow\end{array}$ & Picart et al., 2011 \\
\hline PMMA-g-PEO & EGF & Stromal cells & $\begin{array}{l}\text { Cell proliferation } \uparrow \\
\text { Cell differentiation } \uparrow\end{array}$ & Melarie et al., 2012 \\
\hline Self-assembling peptide-based hydrogels & IGF-1 & MSCs & $\begin{array}{l}\text { Stem cell function } \uparrow \\
\text { Tissue regeneration } \uparrow\end{array}$ & Davis et al. (2006) \\
\hline Hydrogel & $\mathrm{IFN}-\gamma$ & hMSCs & $\begin{array}{l}\text { Immunomodulatory potential } \uparrow \\
\text { Cytokine secretion } \uparrow \\
\text { T cell function } \downarrow\end{array}$ & Gracia et al. (2019) \\
\hline Hyaluronic acid & $\mathrm{N}$-cadherin mimetic peptide (HAV) & MSCs & $\begin{array}{l}\text { Wound healing } \uparrow \\
\text { Chondrogenesis } \uparrow\end{array}$ & Bian et al. (2013) \\
\hline Check polymer & $\mathrm{N}$-cadherin derived peptides & MSCs & $\begin{array}{l}\text { Cell signaling } \uparrow \\
\text { Secretory activities } \uparrow\end{array}$ & Qin et al. (2020) \\
\hline Bi-layered PLGA scaffolds & TGF- $\beta 1$ & Islets & $\begin{array}{l}\text { MCP- } 1 \downarrow \\
\|-12 \downarrow \\
\text { TNF- } \alpha \downarrow \\
\text { Cell engraftment } \uparrow\end{array}$ & Liu et al. (2016a) \\
\hline Oligoethylene glycol SAM & Heparin-binding peptide & HUVECs, MSCS & $\begin{array}{l}\text { Heparin sequestration } \uparrow \\
\text { Cell proliferation } \uparrow\end{array}$ & Hudalla et al. (2011) \\
\hline Hyaluronic acid (HA)-based hydrogels & TGF- $\beta 1$ & CPCs & $\begin{array}{l}\text { Proliferation } \uparrow \\
\text { Differentiation } \uparrow \\
\text { VEGF, HGF } \uparrow\end{array}$ & $\begin{array}{l}\text { Jha et al., } 2015 a \text { and } \\
\text { Jha et al., 2015b }\end{array}$ \\
\hline
\end{tabular}

PLL, poly(L-lysine); PLGA, poly(L-lactide-co-glycolide); IGFBP, insulin-like growth factor-binding protein; SDF-1 $\alpha$, stromal cell-derived factor 1 alpha; BMP-2, bone morphogenetic protein-2; EGF, epidermal growth factor; IGF-1, insulin-like growth factor 1; IFN- $\gamma$, interferon-gamma; TGF- $\beta$, transforming growth factor beta; NPs, nanoparticles; HA, hyaluronic acid; MSCs, mesenchymal stem cells; HUVECs, human umbilical vein endothelial cells; CPCs, cardiac progenitor cells; MCP-1, monocyte chemoattractant protein 1; IL-12, interleukin 12; TNF$\alpha$, tumor necrosis factor alpha; VEGF, vascular endothelial growth factor; HGF, hepatocyte growth factor.

localized presence of cell-instructive cues but also confer enhanced bioactivity to the biomolecules themselves IFN$\gamma$-immobilized hydrogels have been designed to investigate the immunomodulatory function of human mesenchymal stem cells (hMSCs) (Garcia et al., 2019). The tethered form of IFN- $\gamma$ significantly increases the immunomodulatory potential of hMSCs and elevated the secretion of cytokines to halt the activity of $\mathrm{T}$ cells and dendritic cells when compared to the soluble form. The advantages of covalently conjugated IFN- $\gamma$ are likely related to the increased duration of cell licensing, as the tethered form is present throughout the co-culture period, while the unbound IFN- $\gamma$ is washed away rapidly. Moreover, the presence of the tethered form of IFN- $\gamma$ may result in higher local concentrations of IFN- $\gamma$ surrounding the encapsulated hMSCs than the presence of the unbound form. hMSCs encapsulated within hydrogels with tethered IFN- $\gamma$ accelerate the healing of colonic mucosal wounds in both immunocompromised and immunocompetent mice (García et al., 2019). However, future studies are needed to optimize the dose of the tethered biomolecules and decipher the cellular and biological cascades involved so that the efficacy of the therapy can be increased.

It is noteworthy that while ex vivo licensing of hMSCs is therapeutically effective, significant technical, regulatory, and economic issues limit the translational potential of this approach. However, the biomaterial-assisted licensing of hMSCs may greatly enhance their immunomodulatory potential. Although extensive research has been conducted to show biomaterial-assisted tissue regeneration, biomaterials have rarely been exploited for ex vivo licensing of stem cells (Bian et al., 2013; Cosgrove et al., 2016; Li et al., 2017; Zhang et al., 2018b).

To mimic the in vivo microenvironment and promote the chondrogenic differentiation of MSCs in vitro and in vivo, Bian et al. engineered HA hydrogels containing N-cadherin mimetic peptides (Bian et al., 2013). In particular, the His-Ala-Val (HAV) motif present in the first extracellular domain (ECD1) of $\mathrm{N}$-cadherin, which is critical for the homotypic protein interactions that mediate cell-cell adhesion, was engineered into the HA matrix. The tethered HAV motif promoted not only the early chondrogenic differentiation of MSCs and cartilage-specific matrix production in vitro, but also neocartilage formation in implants functionalized with $\mathrm{N}$-cadherin mimetic peptides after subcutaneous implantation. Leckband et al. also evaluated the potential of the full $\mathrm{N}$-cadherin protein, as well as the first two ectodomains of $\mathrm{N}$-cadherin, and a peptide containing the histidine-alanine-valine-aspartic acidvaline (HAVDI) sequence in the first extracellular domain (Qin et al., 2020). While MSCs exhibited comparable rigiditydependent spreading and traction forces, they differed in cell signaling and secretory activities.

The local presentation of bioactive cues at the implantation site may also reduce the inflammatory response by lowering the expression of inflammatory cytokines, thereby reducing graft rejection. In addition, they can be used to investigate the function of encapsulated cells. Liu et al. encapsulated TGF- $\beta$ into a bi-layer scaffold, which not only reduced the expression of 
proinflammatory cytokines, such as monocyte chemoattractant protein 1 (MCP-1), interleukin-12 (IL-12), and tumor necrosis factor-alpha (TNF- $\alpha$ ), but also improved the survival and engraftment of transplanted islet cells in vivo (Liu et al., 2016a). Importantly, TGF- $\beta$-containing scaffolds showed improved glucose levels compared to the control group.

Hudalla et al. conjugated heparin-binding peptide with oligoethylene glycol self-assembling monolayers and reported that the tethered peptide can bind heparin in vitro, thereby sequestering heparin-binding growth factors (Hudalla et al., 2011). Notably, sequestered heparin-bound fibroblast growth factor 2 (FGF-2) enhanced the proliferation of human umbilical vein endothelial cells (HUVECs) and MSCs in vitro. It is expected that such a strategy could also enhance the sequestration of MSC-secreted factors in vivo. Likewise, Heally et al. recently designed cross-linked HA-based hydrogels containing covalently crosslinked heparin and TGF- $\beta 1$, which significantly increased the proliferation, differentiation, and formation of tubular structures in encapsulated cardiac progenitor cells (CPCs) (Jha et al., 2015a; Jha et al., 2015b). Additionally, the levels of several matrix-bound morphogens which significantly contribute to endothelial cell mobilization and vascular invasion, such as insulin-like growth factor-binding proteins (IGFBPs), VEGF, and hepatocyte growth factor (HGF), were significantly increased. This suggests that heparin presentation concentrated and amplified cell-secreted signals to enhance CPC maturation.

\section{DYNAMIC BIOMATERIAL-MEDIATED STEM CELL FATE MODULATION \\ Mechanobiology: A Tool for Cellular Mechanosensing}

It has been widely accepted that cells respond to the ECM via mechanosensing, which drives their migration, proliferation, and differentiation (Discher et al., 2005; Wozniak and Chen, 2009). A myriad of substrates with varying physical properties, such as stiffness, elasticity, and modulus, have been investigated to study the effect of physical parameters on cellular behavior via cellular mechanotransduction. It has been demonstrated that scaffolds with varying stiffness and moduli of elasticity impact cellular behavior differently. Moreover, it has been observed that an increase in the stiffness of cell culture platforms based on collagen-coated polyacrylamide affects the migration, neuronal differentiation, spreading, and stemness of the cells (Pelham and Wang, 1997; Engler et al., 2004; Gilbert et al., 2010).

Despite significant progress in understanding the role of matrix mechanics and elasticity in cellular processes and cellular mechanotransduction, much remains to be investigated. Scientists have discovered new molecules involved in cellular mechanotransduction and have decoupled the effects of material viscoelasticity and viscoplasticity on cellular processes (Table 3) (Marozas et al., 2019). The ECM is not purely elastic, but it also exhibits viscoelastic characteristics; therefore, careful elucidation of both the elastic and viscoelastic components of the ECM in cellular processes is needed (Fung, 1993; McKinnon et al., 2014a; Chaudhuri et al., 2016; Rosales and Anseth, 2016). The distinct role of the viscoelasticity of the matrix on cellular behavior has only recently been studied. While elastic materials store energy, viscoelastic materials can dissipate applied stresses and may undergo stress relaxation in response to deformation and creep in response to them.

Consequently, viscoelastic materials possess elastic and viscous components that affect cellular behavior differently. More specifically, viscoelastic materials undergo two important transitions: contraction in response to applied stress and viscoelastic relaxation when a constant strain is applied. Evaluation of the mechanical properties of most tissues, including brain, adipose, liver, fibrin clot, and fractured hematoma, have shown that they exhibit viscoelastic characteristics (Fung, 1993; McKinnon et al., 2014a; Chaudhuri et al., 2016; Rosales and Anseth, 2016). Consequently, researchers have recently developed biomaterials with varying degrees of viscoelasticity and demonstrated the significant impact of viscoelasticity on cellular behaviors (Chaudhuri et al., 2016; Rosales and Anseth, 2016).

\section{Engineering Biomimetic Platforms for Cellular Mechanosensing}

It is generally understood that in a two-dimensional (2D) microenvironment, cells bind to the matrix through integrins and exert force on it; reciprocally, cells gauge the matrix stiffness via cellular machineries, such as the actin cytoskeleton and molecules that interact with it, such as vinculin and talin. As viscoelastic hydrogels possess stress relaxation and viscoelastic creep, which may dissipate the applied force, cells may, in turn, perceive less stiffness, thereby spreading less. In contrast, in vitro cell culture on 2D collagen-coated polyacrylamide hydrogels resulted in enhanced spreading of MSCs with an increase in the loss modulus or creep (Cameron et al., 2011). Similar results have been found for alginate hydrogels, wherein cells spread more in soft hydrogels than in stiff hydrogels (Chaudhuri et al., 2015). One possible explanation for this observation may be due to increased stability of integrin-ECM bonds that behave as slip bonds owing to stress relaxation. This is because a decrease in the individual bond force leads to an increase in bond lifetime (Chan and Odde, 2008).

The impact of the viscoelasticity of the hydrogels was assessed using 3D cell culture. Just as 2D cell culture influences cellular behavior, 3D culture also significantly impacts cell spreading, proliferation, and differentiation (Das et al., 2016). While synthetic hydrogels are nanoporous, which perturbs cell expansion or volume change during spreading, installing degradable crosslinks in hydrogels partly overcomes this issue. In addition, viscoelastic hydrogels permit cell expansion and volume change (Khetan et al., 2013). Moreover, it has been demonstrated that lowering the stress relaxation time significantly impacts cell spreading (McKinnon et al., 2014a; McKinnon et al., 2014b). Likewise, a reduction in the stress relaxation time promoted the spreading of fibroblasts in RGD-coupled alginate hydrogels (Chaudhuri et al., 2015). 
TABLE 3 | Enhancing stem cell function using instructive biomaterials and mechanotransduction.

\begin{tabular}{|c|c|c|c|}
\hline Biomaterial & Cell/tissue type & Observed effect & References \\
\hline Collagen-based hydrogels & MSCs & Osteogenic differentiation $\uparrow$ & Lou et al. (2018) \\
\hline Hydrogels with tunable dissociation rate constant & MSCs & $\begin{array}{l}\text { Spreading } \uparrow \\
\text { Osteogenic differentiation } \uparrow\end{array}$ & Yang et al. (2021) \\
\hline Hydrogels & MSCs & $\begin{array}{l}\text { alpha-SMA marker } \uparrow \\
\text { Calponin } \uparrow\end{array}$ & Cameron et al. (2011) \\
\hline Alginate-based hydrogels & hMSCs & $\begin{array}{l}\text { Osteogenic differentiation } \uparrow \\
\text { Biomineralization } \uparrow \\
\text { Matrix secretion } \uparrow \\
\text { Cell retention } \uparrow\end{array}$ & Darnell and Mooney, (2017) \\
\hline RGD-PEG-PLA nanoparticles & hMSCs & $\begin{array}{l}\text { Cell retention Cell viability } \uparrow \\
\text { Cell engraftment } \uparrow\end{array}$ & Grosskopf et al. (2020) \\
\hline HA-based hydrogels & ADSCs & Cell retention $\uparrow$ & Andreina Parisi-Amc et al., 2013 \\
\hline PINIPAM-C7-based hydrogels containing YIGSR, IKVAV & Schwann cells & Cell retention $\uparrow$ & Marquardt et al. (2020) \\
\hline PEG-based hydrogels & Chondrocytes & $\begin{array}{l}\text { ECM secretion } \uparrow \\
\text { GAG secretion } \uparrow\end{array}$ & $\begin{array}{l}\text { Richardson et al. (2019) } \\
\text { Feng et al. (2019) }\end{array}$ \\
\hline
\end{tabular}

PINIPAM, poly(N-isopropyl acrylamide); HA, hyaluronic acid; MSCs, mesenchymal stem cells; YIGSR, (Tyr-Ile-Gly-Ser-Arg); IKVAV, (lle-Lys-Val-Ala-Val); ECM, extracellular matrix; GAGs, glycosaminoglycans; $\alpha$-SMA, alpha-smooth muscle actin; PEG, poly(ethylene glycol); hMSCs, human mesenchymal stem cells.

As most cell culture platforms include ECM-derived proteins, such as collagen, gelatin, and Matrigel, the manifested cellular behavior could be partly ascribed to viscoelasticity of these platforms (Nam et al., 2016a; Nam et al., 2016b). Lou et al. demonstrated that interpenetrating hydrogel systems based on HA-crosslinked with dynamic covalent bonds and collagen favored cell spreading and fiber remodeling. More importantly, while most conventional hydrogels are bio-inert, collagen-based hydrogels are not only biocompatible but also recapitulate the fibrillar network, as observed in the ECM microenvironment (Lou et al., 2018). Hydrogels containing more crosslinks promote osteogenic differentiation of the encapsulated cells. Bian et al. demonstrated better spreading and osteogenic differentiation of encapsulated cells in hydrogels with large dissociation rate constants than those of hydrogels with low dissociation rate constants, which was dependent on the concerted action of the cell adhesion structures containing $\beta 1$ integrins (Yang et al., 2021). This indicates that the biofunctionalization of hydrogels also crucial for realizing ECM mimetic effects.

\section{Investigating Cell Fate Using Mechanocompatible Materials}

Varying degrees of stress relaxation and creep have also been reported to affect cellular differentiation. Cameron et al. reported that MSCs cultured on high-creeping hydrogels differentiated into smooth muscle cells (SMCs), which largely expressed SMC markers, such as alpha-smooth muscle actin (a-SMA) and calponin, compared to cells seeded on low-creep hydrogels. In the presence of induction factors, hMSCs cultured on high-creep hydrogels also expressed multiple differentiation capabilities compared to those cultured on low-creep hydrogels (Cameron et al., 2011). It was further demonstrated that hMSCs compensate for decreased passive cytoskeletal tension by spreading via activation of Ras-related C3 botulinum toxin substrate 1 (Rac1), which is a mechanotransductive Rho-GTPase that also plays a role in SMC differentiation.
Mooney et al. also reported the effect of stress relaxation on hMSC differentiation and found that the cells cultured on fastrelaxing alginate-based hydrogels were committed toward the osteogenic lineage in vitro, along with showing higher biomineralization and more matrix secretion than those cultured on slow-relaxing hydrogels (Darnell and Mooney, 2017). More importantly, the implantation of the fast-relaxing hydrogels along with hMSCs led to more bone formation in vivo than that of the control group. Defects implanted with fastrelaxing hydrogels were also filled faster than those implanted with slow-relaxing hydrogels. Examination of the remaining hydrogels in the defect at 2 weeks after implantation revealed that only a small amount of alginate remained in the groups treated with the fast-relaxing hydrogels compared to that of the defects treated with the slow-relaxing hydrogels, which was ascribed to the cellular remodeling of the biomaterials in vivo. These findings encourage further evaluation of stress-relaxing biomaterials for in vivo studies.

\section{Enhancing Stem Cell Survival and Retention Using Mechanocompatible Biomaterials}

Shear-thinning and dynamic hydrogels have enabled long-term survival and retention of transplanted cells in vivo, which is partly attributable to the lesser force experienced by the cells during the injection and the conducive environment provided by the physically or non-covalently crosslinked hydrogels (Figure 3). The Appel group has developed supramolecular injectable polymer nanoparticle-based hydrogels containing RGDtethered polyethylene glycol-poly(L-lactide) (RGD-PEG-PLA), which exhibited shear-thinning and self-healing and significantly improved cell retention as compared to the phosphate buffered saline (PBS) control after subcutaneous implantation in vivo for up to 2 weeks (Grosskopf et al., 2020). Likewise, Heilshorn et al. reported significant retention of transplanted adipose-derived stem cells (ADSCs) using shearthinning and self-healing protein-based hydrogels as assessed 


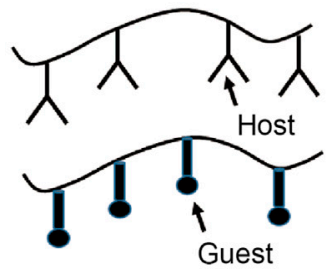

Guest/host polymers

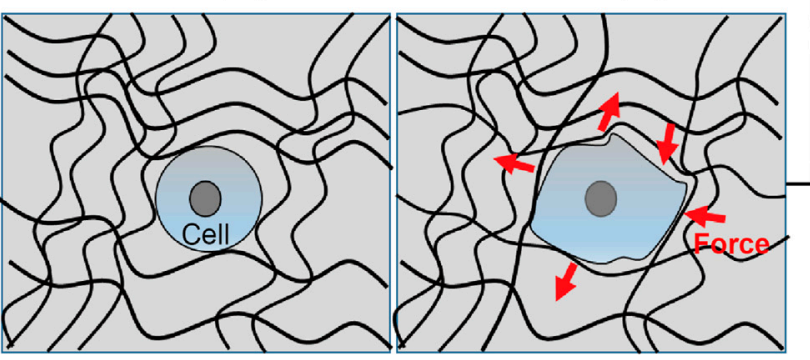

Cells cultured in 3D matrix Cells exert strain on the matrix, magnitude of forces are determined by the initial matrix stiffness

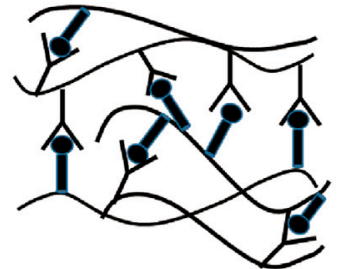

Host-Guest polymers
Forces maintained in the matrix

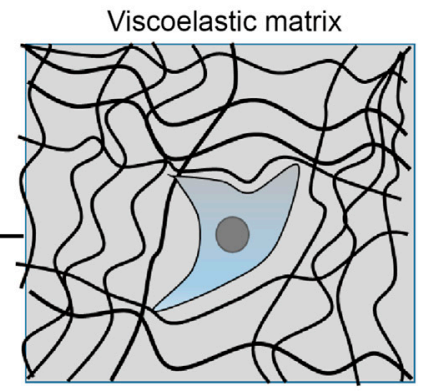

Forces relaxed, cells spread

FIGURE 3 | Interrogating cell function by mechanosensing. Non-covalently crosslinked biomaterials can be engineered to interrogate cell fate by mechanotransduction. Such materials can be designed by installing functional moieties on the individual polymer chains to provide shear-thinning and self-healing characteristics. Once cultured in the elastic or viscoelastic cell substrates, cells can experience different mechanotransduction. In a purely elastic substrate, the cells probe the matrix via integrins and possess spherical shape. On the other hand, if they are cultured on a viscoelastic substrate, both the elastic and viscoelastic components of the matrix can regulate the cell function. Consequently, the cell-mediated forces can be relieved or dissipate in the viscoelastic matrix. The cells can also deform the matrix during spreading, proliferation, and migration.

using bioluminescent imaging (BLI) in vivo for up to 2 weeks (Parisi-Amon et al., 2013). The beneficial effect of the engineered protein-based hydrogels as compared to collagen and alginate is due to the protection of cells during transplantation, instantaneous formation of gels during the injection process, and cell signaling activated by the RGD ligand and hydrogels after transplantation in vivo.

Heilshorn et al. also designed a new variant of shear-thinning and self-healing hydrogels, in which laminin-derived peptide moieties, including pentapeptide, Tyr-Ile-Gly-Ser-Arg (YIGSR), and Ile-LysVal-Ala-Val (IKVAV), were incorporated into the hydrogels. They engineered protein-based supramolecular hydrogels by exploiting weak peptide-peptide interactions between the recombinantly engineered C7 protein and the poly( $\mathrm{N}$-isopropylacrylamide) (PNIPAM) and proline-rich peptide-tethered star-shaped PEGbased hydrogels. Once exploited for the transplantation of Schwann cells (SCs) in a spinal cord injury (SCI) model, these hydrogels remarkably improved cell retention in vivo (740\% higher cell retention vs. saline group) (Marquardt et al., 2020). The enhanced retention of transplanted SCs also led to functional recovery in vivo. Consequently, these hydrogels exhibit great potential for applications in cell biology and regenerative medicine (Wang et al., 2009). Anseth et al. reported significant secretion of ECM proteins and GAGs in PEG-based hydrogels crosslinked by hydrazone bonds (Richardson et al., 2019). At 4 weeks post-encapsulation, the experimental chondrocytes secreted $190 \pm 30 \%$ more collagen and $140 \pm 20 \%$ more sulfated GAGs than those in the control group, which mainly constrained matrix deposition only to the pericellular space.

The same group also investigated the chondroprotective effect of the adaptable hydrazone-crosslinked hydrogels, which displayed a high number of cartilage-specific markers, such as SOX9, and led to the increased deposition of collagen and GAGs. These hydrogels demonstrated an average of $70 \pm 4 \mu \mathrm{g}$ of sulfated GAGs per day and $31 \pm 3 \mu \mathrm{g}$ of collagen per day over 1 month in dynamic compression bioreactors. These types of hydrogels can also be further strengthened by chemical bonds to realize controllable drug release, cellular infiltration, and matrix deposition (Feng et al., 2019). These findings highlight how adaptable hydrogels can enhance regenerative capability, which may have wider implications for regenerative medicine and tissue engineering applications.

\section{ENHANCING STEM CELL RETENTION AND ENGRAFTMENT FOR A FUNCTIONAL TISSUE REPAIR \\ Strategies to Enhance Stem Cell Survival and Retention in vivo}

While MSCs exhibit enormous potential for tissue repair due to their multilineage commitment, their use in clinical settings is 
Biomaterials/Scaffolds/Hydrogel

(Loaded with cells and bioactive cues,

such as RGD, SDF-1 $\alpha$, Substance $P, E 7$ )

$\downarrow$ In-vitro cell culture

Cell-Cell contact $\uparrow$

Cell-ECM contact $\uparrow$

Cell apoptosis $\downarrow$

Cell survival $\uparrow$

$\downarrow \begin{aligned} & \text { In-vivo implantation } \\ & \text { (e.g., cardiac patch, skin patch) }\end{aligned}$

Cell homing $\uparrow$

Immune rejection

Cell engraftment 1

Cell retention $\uparrow$

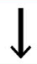

Overall loss of cells $\downarrow$

Cell-cell contact $\uparrow$

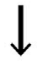

Functional tissue regeneration $\uparrow$

FIGURE 4 | Designing instructive biomaterials for tissue regeneration applications. To ameliorate cell-cell and cell-ECM contacts and impact cellular behavior, instructive biomaterials can be designed by installing an array of functional moieties on biomaterials, such as cell-adhesive ligands like RGD or laminin-derived bioactive cues (IKVAV or YIGSR), the MSCs-affinity peptide "E7", or the EPCs-capturing peptide "TPS". Furthermore, cell inducing/recruiting factors can be incorporated into biomaterials to improve stem cell mobilization, homing, and recruitment in vitro and in vivo. Various cell inducing and recruiting factors, including SDF-1 $\alpha$ and SP have been integrated into biomaterials and shown to promote cell homing and migration. These bioactive cues could be central to ameliorate cell-cell and cell-ECM contacts as well as improve cell survival and retention during tissue regeneration in vivo. Overall, this can lead to functional tissue repair.

hampered partly due to their poor homing to the target site and is further compromised by their poor retention and engraftment at the target site. Therefore, enhancing stem cell homing to the target site as well as improving retention and engraftment may improve the clinical translatability of stem cell therapy. Stem cell transplantation using conventional techniques, such as intravascular or intracardiac injection, leads to the loss of majority of cells, primarily due to poor cell-cell and cell-ECM interactions (Ullah et al., 2015; Lee et al., 2021). Moreover, most of the transplanted cells may lose their immunomodulatory potential. Taken together, these results show the necessity of either preconditioning and licensing therapeutic cells before transplantation or using vehicles for stem cell transplantation (Park et al., 2018). In this section, we discuss recent techniques that have been leveraged to enhance stem cell transplantation, including homing, retention, and engraftment (Figures 4, 5, Table 4).

While transplanted stem cells display poor homing to the target site, certain cytokines and chemokines have been implicated in improving cell homing and could be used to overcome this problem. For instance, SDF-1a has been demonstrated to improve stem cell mobilization, activation, homing, and retention in multiple tissues (Xiang et al., 2020). The SDF-1 $\alpha$-CXCR4 axis has been reported to play a vital role in SDF-1a-mediated cell migration and homing (Petit et al., 2007; Marquez-Curtis and Janowska-Wieczorek, 2013; Shafiq et al., 2017). While hematopoietic stem cells (HSCs) actively express CXCR4, which helps SDF-1a-mediated homing to the bone marrow (BM) as well as transendothelial migration, only a small proportion of MSCs express CXCR4. Consequently, different strategies have been proposed to elevate the expression of CXCR4 in MSCs to improve their trafficking in vitro and in vivo (Teo et al., 2020; Xiang et al., 2020). Xiang et al. leveraged chemotactic microbubble-conjugating SDF-1a, which significantly enhanced the expression of CXCR4 in bone marrow mesenchymal stem cells (BMSCs) during in vitro culture and improved their migration in vitro and in vivo after systematic transplantation in an osteoarthritis rat model (Xiang et al., 2020).

\section{Instructive Biomaterials to Enhance Stem Cell Function in vivo}

Kong et al. designed poly(D, L-lactide-co-glycolide) (PLGA)conjugated HA (PLGA-HA) nanoparticles and encapsulated recombinant SDF-1a protein. PLGA-HA nanoparticles anchored onto MSCs and sustainably released SDF-1 $\alpha$, resulting in an elevated expression of CXCR4 (a 7.0-fold increase compared with untreated MSCs) (Teo et al., 2020). Nanoparticle-mediated release of SDF-1a also improved transendothelial migration in vitro and biodistribution in a hindlimb ischemia model in vivo. Altogether, these results indicate the potential of the SDF-1a-CXCR4 axis in stem cell homing. The same group also leveraged PLGA-HA nanoparticles encapsulating antioxidative cargos (epigallocatechin gallate, EGCG) and manganese oxide $\left(\mathrm{MnO}_{2}\right)$ nanocatalysts) to improve the survival of MSCs in the oxidative tissue microenvironment, which conferred better metabolic and angiogenic potential on the cells (Teo et al., 2020). Similarly, to impact the secretory profile of ADSCs, octadecylamine-grafted HA was complexed onto a liposomal carrier of TNFa, which upregulated the secretion of proangiogenic VEGF and immunomodulatory prostaglandin E2 (PGE2) while decreasing the secretion of antiangiogenic pigment epithelium-derived factors (Leong et al., 2020). TNF-a-tethered ADSCs also promoted vascularization on a chip in vitro as well as perfusion in a murine ischemic hindlimb model in vivo.

Similarly, Parish et al. installed glial cell-derived neurotrophic factor (GDNF) and brain-derived neurotrophic factor (BDNF) on electrospun PCL-based scaffolds to enhance the survival, retention, and engraftment of transplanted neural stem cells (NSCs) (Wang et al., 2016; Nisbet et al., 2018). Both in vitro 


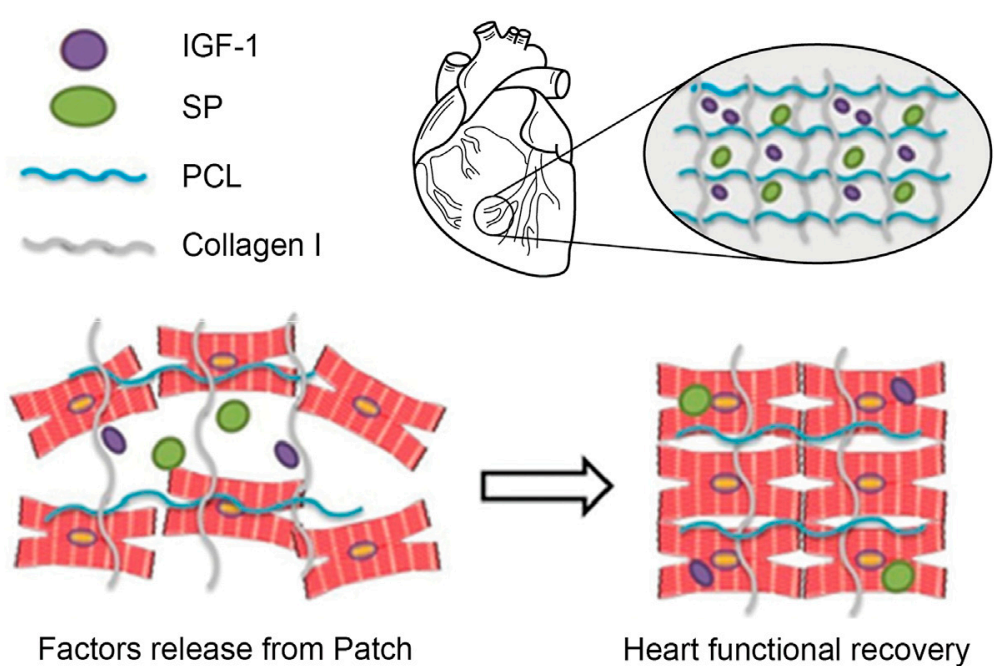

FIGURE 5 | Schematic illustration of MSCs Recruitment in vivo. Schematic diagram of the designed study. Cardiac patches containing SP alone or SP and IGF-1 peptides were fabricated by electrospinning. SP may recruit endogenous stem and progenitor cells, whereas IGF-1 peptide may enhance their retention and engraftment at the target site. Adapted with permission from (Shafiq et al., 2018). Copyrights reserved Oxford Publishers.

and in vivo results showed that the covalently immobilized growth factors enhanced the survival of transplanted NSCs into the targeted tissues. Additionally, GDNF-immobilized scaffolds improved the proliferation and differentiation of NSCs and neurite outgrowth of the transplanted cortical cells while suppressing the number of inflammatory reactive astroglial cells. Nisbet et al. recently embarked on a combination approach for growth factor delivery by blending tissue-specific selfassembling peptide (SAP)-based hydrogels and short nanofibers (Bruggeman et al., 2017). While SAP-based hydrogels provided the rapid release of growth factors, the release started from nanofibers after 6 days in a sustained fashion. These fiber-reinforced hydrogels can be exploited for growth factor delivery and stem cell-mediated tissue repair. Somaa et al. also leveraged brain ECM-mimicking SAP-based hydrogels expressing the laminin-derived epitope "IKVAV" to improve the transplantation of embryonic stem cell (ESC)derived cortical progenitors (Somaa et al., 2017). A network of green fluorescent protein (GFP) was observed in the subcortical nuclei, including the hippocampus, lateral septum, and striatum, as well as in the ventral thalamus and hypothalamus. The cellseeded scaffolds showed potential to improve neuronal function and led to an improvement in motor function over 9 months as compared to the cell- or scaffold-only group (Lee et al., 2018). Cha et al. introduced adhesive protein-based immiscible condensed liquid systems (APICLS) to enhance the survival, retention, and integration of cells with the target tissues (Park et al., 2020c). The APICLS consisted of an immiscible modality from sandcastle worms and an adhesive modality from mussels. Fluid immiscibility may result in the enhanced integration of hydrogels with the myocardium, while adhesiveness may improve the integration of transplanted cells with the host myocardium, concomitantly resulting in enhanced retention and engraftment of the transplanted cells. The hydrogels integrated well with the host myocardium and increased the persistence of the transplanted cells. Secreted paracrine factors released from APICLS with MSCs induced angiogenesis and cardioprotection, and delayed cardiac remodeling, reduced fibrosis, and allowed the recovery of contractive force.

As the loss of cell-cell and cell-ECM interactions leads to reduced survival of transplanted cells, biomaterial-assisted transplantation has been widely pursued to overcome these limitations (Han et al., 2020; Jo et al., 2020). Most importantly, hydrogels fabricated from natural and synthetic polymers have been proposed to improve stem cell transplantation and engraftment into target tissues. Recently, decellularized ECM-based hydrogels have shown promising results compared to conventional hydrogels. For instance, Dvir et al. decellularized omentum tissues and created thermoresponsive omentum ECM-derived hydrogels that induced pluripotent stem cell (iPSC)-derived cardiomyocytes (CMs) (Shevach et al., 2015). CMs were encapsulated in ECM-derived hydrogels using microfluidics. Once injected into the mouse gastrocnemius muscle, these cellular microdroplets showed good survival and retention for up to 2 days.

However, the long-term fate of transplanted cells remains to be elucidated. Successful cell-mediated tissue repair requires the concerted contribution of stem cell transplantation, stem cell viability at the lesion site, and stem cell differentiation into the targeted lineages. Simultaneously enhancing stem cell transplantation and improving the viability and differentiation of the transplanted cells holds great promise for functional tissue repair. This has been realized, at least in soft and hard tissue regeneration. For instance, exogenously transplanted or endogenously recruited stem cells have been directed to differentiate into the targeted lineage, such as osteocytes or chondrocytes, by incorporating chondrogenic growth factors (TGF- $\beta 3$ ) or BMP-2 (Noh et al., 2015; Lee et al., 2017). Similarly, in a seminal study, Song et al. designed poly(organophosphazene) (PPZ)-based thermosensitive hydrogels 
TABLE 4 | Enhancing stem cell retention using instructive biomaterials.

\begin{tabular}{|c|c|c|c|c|}
\hline Biomaterial & Bioactive cue & Cell/tissue type & Observed effect & References \\
\hline PLCL/heparin nanofibers & SDF-1a & & $\begin{array}{l}\text { c-kit positive cells } \uparrow \\
\text { BMSCs } \uparrow \\
\text { Blood vessels } \uparrow\end{array}$ & Shafiq et al. (2017) \\
\hline Microbubbles & SDF-1a & BMSCs & $\begin{array}{l}\text { CXCR4 } \uparrow \\
\text { Cell migration } \uparrow\end{array}$ & Xiang et al. (2020) \\
\hline PLGA/HA NPS & SDF-1a & MSCs & $\begin{array}{l}\text { SDF }-1 \text { a release } \uparrow \\
\text { CXCR } 4 \uparrow \\
\text { Transendothelial cell migration } \uparrow \\
\text { Biodistribution } \uparrow\end{array}$ & Teo et al. (2020) \\
\hline PLGA-HA NPS & EGCG, $\mathrm{MnO}_{2}$ nanocatalysts & MSCs & $\begin{array}{l}\text { Metabolic potential } \uparrow \\
\text { Angiogenesis } \uparrow\end{array}$ & Teo et al. (2020) \\
\hline $\begin{array}{l}\text { Octadecylamine-grafted HA-encapsulating } \\
\text { liposomes }\end{array}$ & $\begin{array}{l}\text { TNF-a-encapsulating } \\
\text { liposomes }\end{array}$ & ADSCs & $\begin{array}{l}\text { VEGF } \uparrow \\
\text { PGE2 } \uparrow \\
\text { Vascularization } \uparrow \\
\text { Pigment epithelium-derived growth } \\
\text { factor } \uparrow\end{array}$ & Leong et al. (2020) \\
\hline PCL scaffolds & GDNF, BDNF & NSCs & $\begin{array}{l}\text { Perfusion } \uparrow \\
\text { Cell survival } \uparrow \\
\text { Cell proliferation } \uparrow \\
\text { Neurites outgrowth } \uparrow\end{array}$ & $\begin{array}{l}\text { Wang et al. (2016) } \\
\text { Nisbet et al. (2018) }\end{array}$ \\
\hline SAP-based hydrogels & GFs & & $\begin{array}{l}\text { Astroglia } \downarrow \\
\text { Growth factors release } \uparrow\end{array}$ & $\begin{array}{l}\text { Bruggeman et al. } \\
\text { (2017) }\end{array}$ \\
\hline APICLS & Paracrine factors & MSCs & $\begin{array}{l}\text { Angiogenesis } \uparrow \\
\text { Cardioprotection } \uparrow \\
\text { Contractile forces } \uparrow \\
\text { Cardiac remodeling } \downarrow \\
\text { Fibrosis } \uparrow\end{array}$ & $\begin{array}{l}\text { Park et al. (2018) } \\
\text { Park et al. (2020a) }\end{array}$ \\
\hline Decellularized omentum tissues & iPSCs-CMs & MSCs & $\begin{array}{l}\text { Cell survival } \uparrow \\
\text { Cell retention } \uparrow\end{array}$ & Gal et al., 2020 \\
\hline PPZ-based hydrogels & $\begin{array}{l}\text { TGF- } \beta \text {-derived peptides } \\
\text { CESPLKRQ \& HAV }\end{array}$ & MSCs & $\begin{array}{l}\text { Cell survival } \uparrow \\
\text { Chondrogenesis } \uparrow\end{array}$ & Hong et al. (2019) \\
\hline Oxygen-preserving hydrogels & HEMA & BMSCs & $\begin{array}{l}\text { Cell survival } \uparrow \\
\text { Cell proliferation dsDNA content of cells } \uparrow\end{array}$ & Niu et al. (2020) \\
\hline PCL/Col-1 nanofibers & $\mathrm{SP}, \mathrm{IGF}-1 \mathrm{C}$ & - & $\begin{array}{l}\text { Cell mobilization } \uparrow \\
\text { Vascularization } \uparrow \\
\text { Cardiac function } \uparrow\end{array}$ & Shafiq et al. (2018) \\
\hline
\end{tabular}

PLCL, poly(L-lactide-co-caprolactone); PLGA, poly(L-lactide-co-glycolide); PCL, polycaprolactone; Col-1, collagen type 1; HA, hyaluronic acid; NPs, nanoparticles; SAP, self-assembling peptides; PPZ, poly(organophosphazene); SDF-1 $\alpha$, stromal cell-derived factor 1 alpha; BDNF, brain-derived neurotrophic factor; GDNF, glial-derived neurotrophic factor; iPSC-CMs, induced pluripotent stem cell-derived cardiomyocytes; TGF- $\beta$, transforming growth factor-beta; MSCs, mesenchymal stem cells; BMSCs, bone marrow mesenchymal stem cells: HEMA, 2-hydroxyethyl methacrylate.

and incorporated a TGF- $\beta 1$-derived peptide sequence (CESPLKRQ) and $\mathrm{N}$-cadherin-derived peptide sequences containing elongated HAV sequences such as CLRAHAVDIN (Hong et al., 2019). More importantly, these chondrogenic peptides were incorporated into the hydrogels by exploiting host-guest interactions by installing $\beta$-cyclodextrin ( $\beta$-CD) on (PPZ) and deriving peptides with adamantane (host). The engineered hydrogels improved stem cell transplantation owing to their injectability and extended viability of the transplanted MSCs, as revealed by the in vivo BLI. The tethered peptide moieties promoted chondrogenesis of transplanted MSCs through mitogen-activated protein kinase (MAPK) pathways. These results suggest that simultaneous approaches can be used to realize MSC-mediated therapeutic benefits.

As most of the transplanted cells are lost due to hypoxia, transplantable cell types are either treated by hypoxic preconditioning or by using oxygen generated in situ. However, stem cell preconditioning as well as the in situ generation of oxygen may not be suitable in many cases because it can result in the overproduction of hydrogen peroxide $\left(\mathrm{H}_{2} \mathrm{O}_{2}\right)$, which may be disadvantageous or cytotoxic to healthy cells. The general method is to incorporate calcium oxide $\left(\mathrm{CaO}_{2}\right)$ or magnesium oxide $\left(\mathrm{MgO}_{2}\right)$ into the tissue culture or hydrogel, which may then react with water to produce $\mathrm{H}_{2} \mathrm{O}_{2}$. The decomposition of $\mathrm{H}_{2} \mathrm{O}_{2}$ yields oxygen. The modification of biomaterials to supply sufficient amounts of oxygen could be a viable option to address the loss of transplanted cells by hypoxia. Guan et al. designed oxygenpreserving hydrogels that enhanced the survival of BMSCs for up to 14 days in an in vitro culture. In contrast, extensive cell death was observed in the control group (Niu et al., 2020).

\section{Instructive Biomaterials to Enhance Function of Extracellular Vesicles}

While a myriad of cell types, including stem cells and pluripotent stem cells, have been demonstrated to improve tissue repair, limited cell retention and engraftment at the target site hampers 
cell therapy. Accordingly, a multitude of biomaterials have been researched to ameliorate the limitations associated with cell therapy, and this has proven to be an effective approach. Stem cell-associated amelioration of the injury microenvironment is mediated by paracrine mechanisms, including the secretion of trophic factors. Because they contain paracrine factors as well as harboring mRNAs and miRNAs, these cell-secreted EVs hold great promise for regenerative medicine and tissue engineering, and have also been used as carriers for drug delivery and tissue repair.

To further expand their use, different types of biomaterialcentered approaches have been proposed to improve the quantity and content of EVs or augment their delivery and retention into target tissue types (Paganini et al., 2019; Iavorovschi and Wang, 2020). For instance, positively charged superparamagnetic iron oxide nanoparticles (SPIONs) encapsulating PLGA-PEI nanoparticles have been shown to cause a significant improvement in the yield of EVs, which was mediated by the autophagy-stimulated Beclin-1 gene (Park et al., 2020a). In addition, exploited bioreactor-mediated mechanical forces improved the yield of exosomes, which was mediated by the mechanosensitivity of yes-associated protein (YAP) mechanosensitivity (Guo et al., 2021). Likewise, the differential response of periodontal stem cell-derived EVs upon culture in three-dimensional magnetically strained culture and, more interestingly, those derived from the strained culture also exhibited better bioactivity in vitro and in vivo when compared to those in a $3 \mathrm{D}$ culture microenvironment ( $\mathrm{Yu}$ et al., 2021). Enhanced expression of miR-133-3p in macrophage migration inhibitory factor (MIF)-engineered umbilical cord MSC (ucMSC)-derived EVs was observed compared to the control umMSCs group (Zhu et al., 2021). Similarly, stimulating human placental-derived MSCs using NO-releasing polymers has been shown to have a significantly improved angiogenic effect on HUVECs, mediated by an increased expression of VEGF and miR-126. However, due to overlapping size range and the lack of specific markers, current EVs preparations, including exosomes are highly heterogenous with undefined purity. International Society for Extracellular Vesicles (ISEV) has introduced guidelines/recommendations for the minimal information for studies of EVs (MISEV) regarding the nomenclature, isolation, and characterization of EVs, which outlines an exhaustive platform for EV related research (Thery et al., 2018).

In addition to improving the quantity and content of EVs, enhancing their retention and sustained release at the injury site also holds great promise, which has been addressed by exploiting different types of strategies, including immobilization on scaffolds or encapsulation into hydrogels. For instance, Li et al. leveraged integrin-ligand interactions to immobilize MSC-derived EVs on HA-based hydrogels for degenerated nerve regeneration, and the immobilized EVs exhibited greater retention and sustained release into nerve tissues. This not only improved motor function and urinary tissue preservation but also mitigated inflammation and oxidation ( $\mathrm{Li}$ et al., 2020). Because the majority of intravenously administered EVs are adsorbed within the liver, biomaterial-mediated strategies can be exploited to improve their homing to the target tissues. Recently, EVs covalently conjugated with cardiac homing peptide (CHP, CSTSMLKAC) have shown a considerably improved homing effect to infarcted myocardium, which resulted in improved cardiac function and reduced fibrosis (Vandergriff et al., 2018).

Chemical immobilization of the targeting ligand is an interesting avenue, but harsh chemical conjugation chemistries can hamper this approach. Biorthogonal labeling of EVs during their secretion from the cell in the presence of azide-containing moieties followed by alkyne-azide click conjugation of target proteins, such as biotin, improved cellular uptake of EVs as well as the delivery of target loads into cells (Wang et al., 2015). EVs with magnetoresponsive ability have been further developed by employing oleic-acid modified iron oxide nanoparticles, which exhibit magnetic field-mediated homing to adipose tissue-derived stem cells (ADSCs) (Mizuta et al., 2019). To further leverage EVs with the potential to evade macrophage uptake and improve their cellular internalization, the platelet membrane may be anchored onto EVs. Platelet membrane hybridized EVs have exhibited macropinocytosismediated cellular internalization as well as distinct myocardial homing compared to unmodified EVs (Hu et al., 2021).

In addition to their modification, polymeric carriers have been designed to allow the controlled release of EVs. For instance, a PLGA copolymer was used to deliver odontogenic EVs, which helped to regenerate dentin (Swanson et al., 2020) and the introduction of phage-display derived "LLP2A" ligand capable of interacting with the $\alpha 4 \beta 1$ integrins was utilized to immobilize EVs on electrospun scaffolds (Hao et al., 2020). Similarly, tannic acid-mediated coupling of EVs on polyetheretherketone (PEEK)based implants resulted in the controlled release of EVs, osteoimmunomodulation-mediated angiogenesis, and new bone formation (Fan et al., 2021). Alginate hydrogels have also been exploited to improve retention and achieve controlled release of dendritic cell (DC)-derived EVs, which induced the recruitment of regulatory $\mathrm{T}$ cells (Treg), shifted macrophages to reparative M2 phenotypes, and improved cardiac function (Zhang et al., 2021). Moreover, an array of biomaterials and scaffolds has been used to achieve the sustained and localized release of EVs for tissue repair (Liu et al., 2017a; Zhang et al., 2018a; Zhou et al., 2019; Brennan et al., 2020).

\section{MODULATING THE INJURY MICROENVIRONMENT TO ENHANCE STEM CELL RETENTION AND ENGRAFTMENT}

Because biomaterial-mediated inflammation of the injury microenvironment also leads to impaired tissue repair and implant failure, modulation of the injury microenvironment may have great potential as a way of improving the engraftment of transplanted cells and functional repair. A promising approach to modulating the injury microenvironment is to release inflammation-resolving biomolecules from the implants or sequester endogenous 


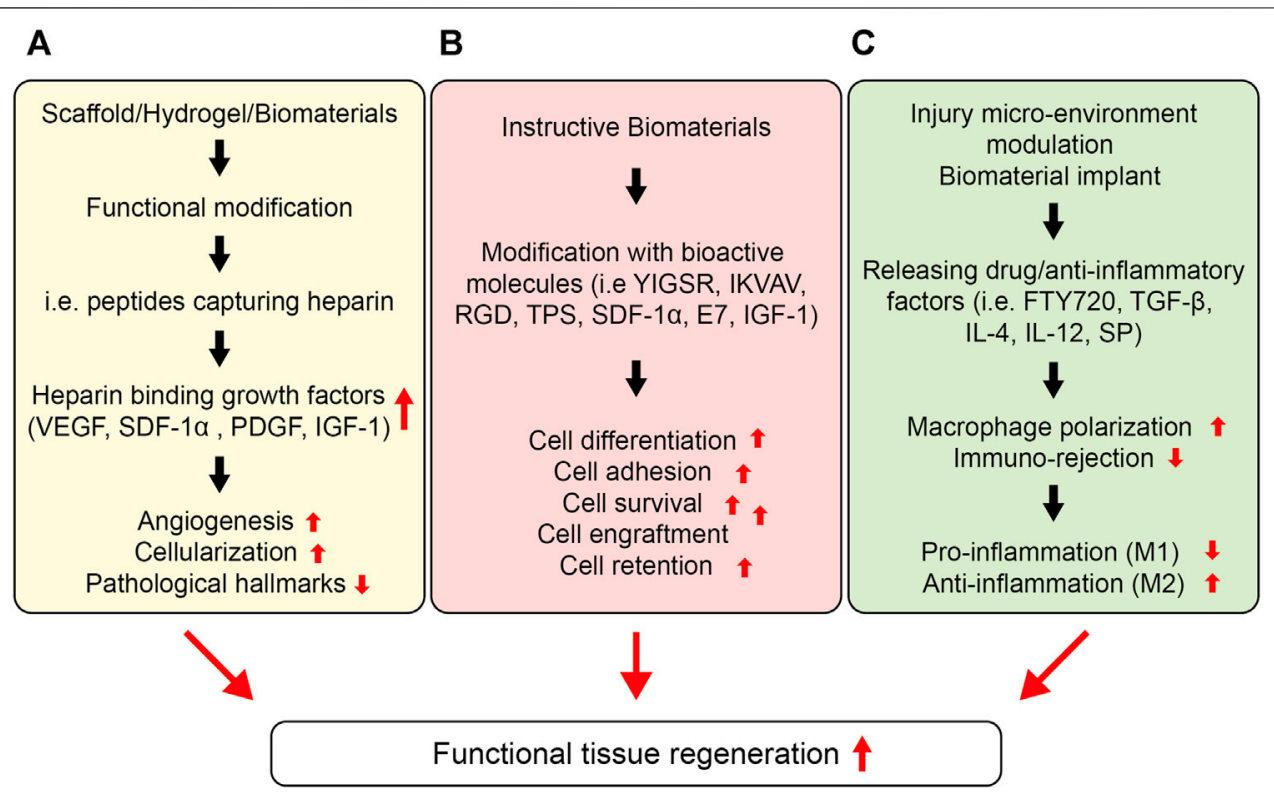

FIGURE 6 | Strategies to enhance cell function for a functional tissue repair. (A) Stem cell function can be improved by sequestering proteins in situ via the functional design of biomaterials. Different types of peptides could be installed into biomaterials with the ability to endogenously capture cell-signaling proteins, such as heparin, which can then bind several heparin-binding growth factors, such as, VEGF, PDGF, IGF etc. (B) Instructive biomaterials designed by incorporating bioactive cues can also enhance cell survival, retention, and engraftment. Depending upon the nature of the signaling cue, they can enhance the stem cell differentiation, proliferation, or migration in vitro and in vivo, which can be central to the formation of in vitro tissue-engineered constructs as well as enhance cell engraftment in vivo. (C) The injury microenvironment can also be tuned to ameliorate stem cell function, including survival, retention, and engraftment. This can be achieved by releasing anti-inflammatory therapeutics and cytokines, including IL-4, IL-1, TGF- $\beta 1$, rapamycin, resveratrol, SP etc. Consequently, these therapeutics and functional cues can lower the proinflammatory response and enhance the anti-inflammatory response overcoming the immune rejection, lowering the fibrosis, and enhancing the stem cell survival and retention as well as engraftment at the targeted site.

morphogens to ameliorate the tissue repair process (Figure 6 and Table 5). For instance, Gower et al. assembled TGF- $\beta$ into layer-bylayer (LBL) scaffolds to resolve inflammation in the injury microenvironment and enhance the survival and retention of transplanted islets (Liu et al., 2016b). The localized release of TGF- $\beta$ resulted in lower expression of inflammatory cytokines, such as TNF- $\alpha$, IL-12, and MCP-1, thereby causing a significant decrease in leukocyte recruitment and higher survival of the transplanted islets as compared to those of the control group. Consequently, biomaterial-mediated modulation of the immune response has considerable potential to enhance cell function in cell-based therapies. Similarly, Cheng et al. leveraged microneedle patches to deliver cardiac stem cells (CSCs) to the heart (Tang et al., 2018). The microneedle patch was fabricated from polyvinyl alcohol (PVA), which provided a conducive environment for the survival of the transplanted CSCs and leveraged a pathway for the exchange of nutrients with the heart and the transportation of paracrine factors from the transplanted cells, thereby leading to better regeneration of the infarcted heart. Consequently, the cells transplanted using microneedle patches reduced scar tissue formation, increased blood vessel regeneration, and left ventricular wall stabilization in the infarcted heart. This technique could be further tailored to release paracrine factors in response to the injury microenvironment.

To further tailor the injury microenvironment and improve the survival and engraftment of the transplanted cells, Duvall et al. designed reactive oxygen species (ROS)-scavenging hydrogels by installing dithiol polymers into PEG-maleimide-based hydrogels, which act as antioxidative biomaterials that quench ROS and enhance the survival of the transplanted cells (Martin et al., 2020). ROS-degradable poly (thioketal) (PTK) polymers were designed and leveraged as platforms for MSC transplantation. ROS-sensitive crosslinks enhanced the survival of MSCs by scavenging free radicals, and the transplanted cells showed significantly higher survival when this platform was used than when platforms containing only PEG hydrogels and enzymatically degradable hydrogels. These types of oxidationsensitive biomaterials may afford novel hydrogels for modulating the injury microenvironment to benefit cell transplantation and drug delivery. Because hypoxia and nutrient deficiency cause cell loss upon transplantation and require strategies to improve the survival of the transplanted islets at the implantation site, Desai et al. fabricated an immunoprotective device capable of providing a sustained release of amino acids, namely, alanine and glutamine, which significantly improved the survival of the islets under nutrient-limiting conditions in vitro as well as in the subcutaneous space in vivo by conferring immune protection on the cells (Chendke et al., 2019). Amino acids were released from the films for up to 18 days. Grafts co-releasing alanine and glutamine increased cell survival in vivo by $33 \%$ compared to the control grafts, which lacked amino acids and showed $0 \%$ survival of the cells at 21 days post-transplantation. In another study, Wang et al. anchored immunosuppressant-releasing nanoparticles to hydrogels ( $\mathrm{Li}$ et al., 2018). Tacrolimus was investigated as an immunosuppressant and bound to RADA16-modified PLGA nanoparticles (NPs). 
TABLE 5 | Enhancing stem cell function by modulating the injury microenvironment.

\begin{tabular}{|c|c|c|c|}
\hline Biomaterial & Cell/tissue type & Observed effect & References \\
\hline TGF- $\beta$-loaded Layer-by-layer scaffolds & Islets & $\begin{array}{l}\text { Cell survival } \uparrow \\
\text { Inflammatory cytokines (e.g., TNF- } \alpha, \mathrm{IL}-12 \text {, } \\
\text { MCP-1) } \downarrow \\
\text { Leukocytes } \downarrow\end{array}$ & Liu et al. (2016b) \\
\hline Poly (vinyl alcohol) microneedle patch & CSCs & $\begin{array}{l}\text { Paracrine factors secretion } \uparrow \\
\text { Scar tissue } \downarrow \\
\text { Blood vessel regeneration } \uparrow\end{array}$ & Tang et al., 2020 \\
\hline PEG-maleimide hydrogels & MSCs & $\begin{array}{l}\text { ROS scavenging } \uparrow \\
\text { Cell survival } \uparrow\end{array}$ & Martin et al. (2020) \\
\hline Immuno-protective device eluting alanine and glutamine & Islets & $\begin{array}{l}\text { Cell survival } \uparrow \\
\text { Immuno-protection of cells } \uparrow\end{array}$ & $\begin{array}{l}\text { Chendke et al. } \\
\text { (2019) }\end{array}$ \\
\hline Hydrogels encapsulating tacrolimus-eluting PLGA NPs & EPCs & $\begin{array}{l}\text { Cell survival } \uparrow \\
\text { Blood perfusion } \uparrow\end{array}$ & Li et al. (2018) \\
\hline $\begin{array}{l}\text { Self-assembling peptides based hydrogels containing laminin- } \\
\text { derived IKVAV motif }\end{array}$ & $\begin{array}{l}\text { ESCs-derived cortical } \\
\text { progenitors }\end{array}$ & $\begin{array}{l}\text { Neuronal function } \uparrow \\
\text { Motor function } \uparrow\end{array}$ & Somaa et al. (2017) \\
\hline
\end{tabular}

TGF- $\beta$, transforming growth factor beta; PEG, poly(ethylene glycol); PLGA, poly(L-lactide-co-glycolide); NPs, nanoparticles; CSCs, cardiac stem cells; ESCs, embryonic stem cells; MSCs, mesenchymal stem cells; EPCs, endothelial progenitor cells; TNF- $\alpha$, tumor necrosis factor alpha; MCP-1, monocyte chemoattractant protein 1; IL-12, interleukin 12.

Tacrolimus was released for up to 28 days, which increased the survival of endothelial progenitor cells (EPCs) in vitro and in vivo. The NP-mediated release of tacrolimus lessened immune rejection; consequently, transplanted EPCs improved blood perfusion in a hind limb ischemia model. Shafiq et al. designed cardiac patches codelivering neuropeptide substance $\mathrm{P}$ and insulin-like growth factor-1 domain C (IGF-1C) and evaluated their potential in an infarcted myocardium model in mice. IGF-1C was used to provide a cardioprotective environment to cardiomyocytes, which could reduce their apoptosis following myocardial infarction. The results showed that SP and IGF-1C acted synergistically to improve endogenous stem cell and progenitor cell recruitment and lowered the frequency of apoptosis in vivo (Shafiq et al., 2018) (Figure 5).

\section{CONCLUSION AND PERSPECTIVES}

Stem cell transplantation has received considerable attention from researchers. However, it is often accompanied by reduced survival, retention, and engraftment resulting from poor cell-cell and cell-ECM interactions during cell administration and post-transplantation. Consequently, to avoid cell loss during transplantation, many biomaterial platforms have been proposed, such as natural and synthetic polymer-based hydrogels, micro-and nano-spheres, and intelligent scaffolds. This work has substantially improved our understanding of the cell-cell and cell-matrix interactions as well as resulted in better cell survival in vitro and during transplantation (Figure 6). A wide variety of bioactive cues have been incorporated into biomaterials to modulate stem cell behavior in vitro and in vivo. Such bioactive cues not only help improve cell survival and growth during in vitro culture, but also stimulate cellular behavior post-transplantation. As cells perceive a gradient of morphogens and signaling cues in vivo, which may be tethered to the ECM or exist in soluble form, proper knowledge of these bioactive cues is necessary to understand cellular behavior. Several ECM-derived peptide sequences have been installed in biomaterials to affect cellular behavior in vitro and in vivo. Likewise, phage-display technology has garnered attention to screen peptides for modulating cellular fate. Screening biomaterials also holds great promise for providing cell-instructive platforms. The spatiotemporal presentation of signaling cues is also of fundamental importance, and has been studied by installing tissue microenvironment-responsive sequences into biomaterials to leverage timely cues to the transplanted cells and improve their survival, retention, and engraftment in the targeted tissues. Engineering biomaterials to leverage bioactive cues in a spatiotemporal fashion holds great promise and will require further attention from researchers.

The ECM helps instruct cell fate through tethered and soluble bioactive cues as well as mechanotransduction pathways. It has been determined that ECM stiffness regulates cell fate and drives tissuespecific cell lineage commitment. As the ECM exhibits non-linear mechanical behavior encompassing elastic and viscoelastic components, biomaterials that mimic the mechanical behavior of the ECM hold great promise for cell fate modulation and tissue engineering applications. Future research is therefore warranted to identify and develop such biomaterials, which may not only improve our understanding of cellular mechanotransduction but also leverage mechanotransduction pathways to discern development and pathological mechanisms. Interdisciplinary knowledge about the development of such biomaterials is needed to provide cellinstructive platforms.

The preconditioning and licensing of cells may help improve survival and engraftment after transplantation. As a library of growth factors that can drive tissue-specific cell lineage commitment has already been identified and that can be incorporated into biomaterials to improve cell survival and engraftment of the targeted tissues, a precise understanding of cell fate in vivo and cell engraftment into the targeted tissue is of pivotal significance. This has been realized by the integration of traceable magnetic nanoparticles and probes. As discussed above, cells perceive ECM-derived signaling cues in a continuum fashion by soluble and tethered ligands, and the judicious selection of biomaterials leveraging the on-off switch of 
signaling cues may help further investigate the cellular fate in vitro and in vivo. The identification of biomaterial platforms harboring and leveraging signaling cues in a cellmediated fashion holds great promise. Lastly, because the fate of the transplanted cells is also governed by the injury or transplantation microenvironment and most of the transplanted cells are lost due to immune rejection, immune protection of the transplanted cells at the injury site may help improve cell survival and retention at the target site as well as their engraftment post-transplantation. This has been achieved by delivering immune-protective cues from biomaterial platforms in a spatiotemporal manner.

\section{AUTHOR CONTRIBUTIONS}

MS, OA, SH, and DK co-wrote the manuscript; DK supervised the project.

\section{REFERENCES}

Abdulghani, S., and Mitchell, G. R. (2019). Biomaterials for In Situ Tissue Regeneration: A Review. Biomolecules 9, 750. doi:10.3390/biom9110750

Bian, L., Guvendiren, M., Mauck, R. L., and Burdick, J. A. (2013). Hydrogels that Mimic Developmentally Relevant Matrix and N-Cadherin Interactions Enhance MSC Chondrogenesis. Proc. Natl. Acad. Sci. 110, 10117-10122. doi:10.1073/pnas. 1214100110

Brennan, M. Á., Layrolle, P., and Mooney, D. J. (2020). Biomaterials Functionalized with MSC Secreted Extracellular Vesicles and Soluble Factors for Tissue Regeneration. Adv. Funct. Mater. 30, 1909125. doi:10.1002/adfm.201909125

Bruggeman, K. F., Wang, Y., Maclean, F. L., Parish, C. L., Williams, R. J., and Nisbet, D. R. (2017). Temporally Controlled Growth Factor Delivery from a Self-Assembling Peptide Hydrogel and Electrospun Nanofibre Composite Scaffold. Nanoscale 9, 13661-13669. doi:10.1039/c7nr05004f

Cameron, A. R., Frith, J. E., and Cooper-White, J. J. (2011). The Influence of Substrate Creep on Mesenchymal Stem Cell Behaviour and Phenotype. Biomaterials 32, 5979-5993. doi:10.1016/j.biomaterials.2011.04.003

Cameron, D. J. A., and Shaver, M. P. (2011). Aliphatic Polyester Polymer Stars: Synthesis, Properties and Applications in Biomedicine and Nanotechnology. Chem. Soc. Rev. 40, 1761-1776. doi:10.1039/c0cs00091d

Chan, C. E., and Odde, D. J. (2008). Traction Dynamics of Filopodia on Compliant Substrates. Science 322, 1687-1691. doi:10.1126/science.1163595

Chaudhuri, O., Gu, L., Darnell, M., Klumpers, D., Bencherif, S. A., Weaver, J. C., et al. (2015). Substrate Stress Relaxation Regulates Cell Spreading. Nat. Commun. 6, 6364. doi:10.1038/ncomms7365

Chaudhuri, O., Gu, L., Klumpers, D., Darnell, M., Bencherif, S. A., Weaver, J. C., et al. (2016). Hydrogels with Tunable Stress Relaxation Regulate Stem Cell Fate and Activity. Nat. Mater 15, 326-334. doi:10.1038/nmat4489

Chen, T. T., Luque, A., Lee, S., Anderson, S. M., Segura, T., and Iruela-Arispe, M. L. (2010). Anchorage of VEGF to the Extracellular Matrix Conveys Differential Signaling Responses to Endothelial Cells. J. Cel Biol 188, 595-609. doi:10.1083/ jcb.200906044

Chendke, G. S., Faleo, G., Juang, C., Parent, A. V., Bernards, D. A., Hebrok, M., et al. (2019). Supporting Survival of Transplanted Stem-Cell-Derived Insulin-Producing Cells in an Encapsulation Device Augmented with Controlled Release of Amino Acids. Adv. Biosys. 3, 1900086. doi:10.1002/adbi.201900086

Cosgrove, B. D., Mui, K. L., Driscoll, T. P., Caliari, S. R., Mehta, K. D., Assoian, R. K., et al. (2016). N-cadherin Adhesive Interactions Modulate Matrix Mechanosensing and Fate Commitment of Mesenchymal Stem Cells. Nat. Mater 15, 1297-1306. doi:10.1038/nmat4725

Crouzier, T., Fourel, L., Boudou, T., Albigès-Rizo, C., and Picart, C. (2011). Presentation of BMP-2 from a Soft Biopolymeric Film Unveils its Activity

\section{FUNDING}

DK was supported by National Research Foundation of Korea (NRF-2019R1A2C2004437 and 2020R1A4A3079755) and the MSIT (Ministry of Science and ICT), Korea, under the ICT Creative Consilience program (IITP-2020-0-01819) supervised by the IITP (Institute for Information and communications Technology Planning and Evaluation).

\section{ACKNOWLEDGMENTS}

Authors thank members of the Applied Mechanobiology Group at the Korea University for thoughtful discussion regarding the mechanobiology of stem cells. This work was supported by KU-KIST Graduate School of Converging Science and Technology Program, National Research Foundation of Korea, and Ministry of Science and ICT.

on Cell Adhesion and Migration. Adv. Mater. 23, H111-H118. doi:10.1002/ adma.201004637

Darnell, M., and Mooney, D. J. (2017). Leveraging Advances in Biology to Design Biomaterials. Nat. Mater 16, 1178-1185. doi:10.1038/nmat4991

Das, R. K., Gocheva, V., Hammink, R., Zouani, O. F., and Rowan, A. E. (2016). Stress-stiffening-mediated Stem-Cell Commitment Switch in Soft Responsive Hydrogels. Nat. Mater 15, 318-325. doi:10.1038/nmat4483

Davis, M. E., Hsieh, P. C. H., Takahashi, T., Song, Q., Zhang, S., Kamm, R. D., et al. (2006). Local Myocardial Insulin-like Growth Factor 1 (IGF-1) Delivery with Biotinylated Peptide Nanofibers Improves Cell Therapy for Myocardial Infarction. Proc. Natl. Acad. Sci. 103, 8155-8160. doi:10.1073/pnas.0602877103

Discher, D. E., Janmey, P., and Wang, Y.-L. (2005). Tissue Cells Feel and Respond to the Stiffness of Their Substrate. Science 310, 1139-1143. doi:10.1126/ science. 1116995

Engler, A. J., Griffin, M. A., Sen, S., Bönnemann, C. G., Sweeney, H. L., and Discher, D. E. (2004). Myotubes Differentiate Optimally on Substrates with Tissue-like Stiffness. J. Cel Biol 166, 877-887. doi:10.1083/jcb.200405004

Fan, L., Guan, P., Xiao, C., Wen, H., Wang, Q., Liu, C., et al. (2021). Exosomefunctionalized Polyetheretherketone-Based Implant with Immunomodulatory Property for Enhancing Osseointegration. Bioactive Mater. 6, 2754-2766. doi:10.1016/j.bioactmat.2021.02.005

Fan, V. H., Au, A., Tamama, K., Littrell, R., Richardson, L. B., Wright, J. W., et al. (2007). Tethered Epidermal Growth Factor Provides a Survival Advantage to Mesenchymal Stem Cells. Stem Cells 25, 1241-1251. doi:10.1634/ stemcells.2006-0320

Feng, P., Yang, H., Ding, H., Lin, H., Chen, W., and Chou, K.-C. (2019). iDNA6mA-PseKNC: Identifying DNA N6-Methyladenosine Sites by Incorporating Nucleotide Physicochemical Properties into PseKNC. Genomics 111, 96-102. doi:10.1016/j.ygeno.2018.01.005

Freudenberg, U., Liang, Y., Kiick, K. L., and Werner, C. (2016). Glycosaminoglycan-Based Biohybrid Hydrogels: A Sweet and Smart Choice for Multifunctional Biomaterials. Adv. Mater. 28, 8861-8891. doi:10.1002/ adma.201601908

Fung, Y.-C. (1993). Mechanical Properties of Living Tissues. Springer.

García, J. R., Quirós, M., Han, W. M., O’leary, M. N., Cox, G. N., Nusrat, A., et al. (2019). IFN- $\gamma$-tethered Hydrogels Enhance Mesenchymal Stem Cell-Based Immunomodulation and Promote Tissue Repair. Biomaterials 220, 119403. doi:10.1016/j.biomaterials.2019.119403

García, J. R., Quirós, M., Han, W. M., O’leary, M. N., Cox, G. N., Nusrat, A., et al. (2019). IFN- $\gamma$-tethered Hydrogels Enhance Mesenchymal Stem Cell-Based Immunomodulation and Promote Tissue Repair. Biomaterials 220, 119403. doi:10.1016/j.biomaterials.2019.119403

Gilbert, P. M., Havenstrite, K. L., Magnusson, K. E. G., Sacco, A., Leonardi, N. A., Kraft, P., et al. (2010). Substrate Elasticity Regulates Skeletal Muscle Stem Cell Self-Renewal in Culture. Science 329, 1078-1081. doi:10.1126/science.1191035 
Grosskopf, A. K., Roth, G. A., Smith, A. A. A., Gale, E. C., Hernandez, H. L., and Appel, E. A. (2020). Injectable Supramolecular Polymer-Nanoparticle Hydrogels Enhance Human Mesenchymal Stem Cell Delivery. Bioeng. Transl Med. 5, e10147. doi:10.1002/btm2.10147

Guo, S., Debbi, L., Zohar, B., Samuel, R., Arzi, R. S., Fried, A. I., et al. (2021). Stimulating Extracellular Vesicles Production from Engineered Tissues by Mechanical Forces. Nano Lett. 21, 2497-2504. doi:10.1021/ acs.nanolett.0c04834

Han, S. B., Kim, J. K., Lee, G., and Kim, D. H. (2020). Mechanical Properties of Materials for Stem Cell Differentiation. Adv. Biosyst. 4, e2000247. doi:10.1002/ adbi.202000247

Hao, D., Swindell, H. S., Ramasubramanian, L., Liu, R., Lam, K. S., Farmer, D. L., et al. (2020). Extracellular Matrix Mimicking Nanofibrous Scaffolds Modified with Mesenchymal Stem Cell-Derived Extracellular Vesicles for Improved Vascularization. Front. Bioeng. Biotechnol. 8, 633. doi:10.3389/fbioe.2020.00633

Hettiaratchi, M. H., Guldberg, R. E., and Mcdevitt, T. C. (2016). Biomaterial Strategies for Controlling Stem Cell Fate via Morphogen Sequestration. J. Mater. Chem. B 4, 3464-3481. doi:10.1039/c5tb02575c

Hong, K. H., Kim, Y. M., and Song, S. C. (2019). Fine-Tunable and Injectable 3D Hydrogel for On-Demand Stem Cell Niche. Adv. Sci. 6, 1900597. doi:10.1002/ advs. 201900597

Hu, S., Wang, X., Li, Z., Zhu, D., Cores, J., Wang, Z., et al. (2021). Platelet Membrane and Stem Cell Exosome Hybrids Enhance Cellular Uptake and Targeting to Heart Injury. Nano Today 39, 101210. doi:10.1016/ j.nantod.2021.101210

Hudalla, G. A., Kouris, N. A., Koepsel, J. T., Ogle, B. M., and Murphy, W. L. (2011). Harnessing Endogenous Growth Factor Activity Modulates Stem Cell Behavior. Integr. Biol. 3, 832-842. doi:10.1039/c1ib00021g

Iavorovschi, A. M., and Wang, A. (2020). Engineering Mesenchymal Stromal/stem Cell-Derived Extracellular Vesicles with Improved Targeting and Therapeutic Efficiency for the Treatment of central Nervous System Disorders. Neural Regen. Res. 15, 2235-2236. doi:10.4103/1673-5374.284982

Jha, A. K., Mathur, A., Svedlund, F. L., Ye, J., Yeghiazarians, Y., and Healy, K. E. (2015a). Molecular Weight and Concentration of Heparin in Hyaluronic AcidBased Matrices Modulates Growth Factor Retention Kinetics and Stem Cell Fate. J. Controlled Release 209, 308-316. doi:10.1016/j.jconrel.2015.04.034

Jha, A. K., Tharp, K. M., Ye, J., Santiago-Ortiz, J. L., Jackson, W. M., Stahl, A., et al. (2015b). Enhanced Survival and Engraftment of Transplanted Stem Cells Using Growth Factor Sequestering Hydrogels. Biomaterials 47, 1-12. doi:10.1016/ j.biomaterials.2014.12.043

Jo, J., Abdi Nansa, S., and Kim, D. H. (2020). Molecular Regulators of Cellular Mechanoadaptation at Cell-Material Interfaces. Front. Bioeng. Biotechnol. 8, 608569. doi:10.3389/fbioe.2020.608569

Karantalis, V., and Hare, J. M. (2015). Use of Mesenchymal Stem Cells for Therapy of Cardiac Disease. Circ. Res. 116, 1413-1430. doi:10.1161/ circresaha.116.303614

Khetan, S., Guvendiren, M., Legant, W. R., Cohen, D. M., Chen, C. S., and Burdick, J. A. (2013). Degradation-mediated Cellular Traction Directs Stem Cell Fate in Covalently Crosslinked Three-Dimensional Hydrogels. Nat. Mater 12, 458-465. doi:10.1038/nmat3586

Lee, C.-F., Li, Z.-Y., Ho, P. T. P., Hirano, N., Zhang, Q., and Shang, H. (2017). Formation and Atmosphere of Complex Organic Molecules of the HH 212 Protostellar Disk. Astrophysical J. 843, 27. doi:10.3847/1538-4357/aa7757

Lee, G., Han, S. B., and Kim, D. H. (2021). Cell-ECM Contact-Guided Intracellular Polarization Is Mediated via Lamin A/C Dependent Nucleus-Cytoskeletal Connection. Biomaterials 268, 120548. doi:10.1016/j.biomaterials.2020.120548

Lee, G., Lim, J., Park, J., Lee, W., Yoon, D. S., Kim, S. H., et al. (2018). Construction of Neurospheroids via Surface Modified Concave Microwells. J. Ind. Eng. Chem. 62, 341-351. doi:10.1016/j.jiec.2018.01.014

Leong, J., Hong, Y.-T., Wu, Y.-F., Ko, E., Dvoretskiy, S., Teo, J. Y., et al. (2020). Surface Tethering of Inflammation-Modulatory Nanostimulators to Stem Cells for Ischemic Muscle Repair. ACS Nano 14, 5298-5313. doi:10.1021/ acsnano.9b04926

Li, L., Zhang, Y., Mu, J., Chen, J., Zhang, C., Cao, H., et al. (2020). Transplantation of Human Mesenchymal Stem-Cell-Derived Exosomes Immobilized in an Adhesive Hydrogel for Effective Treatment of Spinal Cord Injury. Nano Lett. 20, 4298-4305. doi:10.1021/acs.nanolett.0c00929
Li, R., Liang, J., He, Y., Qin, J., He, H., Lee, S., et al. (2018). Sustained Release of Immunosuppressant by Nanoparticle-Anchoring Hydrogel Scaffold Improved the Survival of Transplanted Stem Cells and Tissue Regeneration. Theranostics 8, 878-893. doi:10.7150/thno.22072

Li, R., Xu, J., Wong, D. S. H., Li, J., Zhao, P., and Bian, L. (2017). Self-assembled $\mathrm{N}$-Cadherin Mimetic Peptide Hydrogels Promote the Chondrogenesis of Mesenchymal Stem Cells through Inhibition of Canonical Wnt $/ \beta$-Catenin Signaling. Biomaterials 145, 33-43. doi:10.1016/j.biomaterials.2017.08.031

Liu, J., Chen, S., Wang, W., Ning, B.-F., Chen, F., Shen, W., et al. (2016a). Cancerassociated Fibroblasts Promote Hepatocellular Carcinoma Metastasis through Chemokine-Activated Hedgehog and TGF- $\beta$ Pathways. Cancer Lett. 379, 49-59. doi:10.1016/j.canlet.2016.05.022

Liu, J. M. H., Zhang, J., Zhang, X., Hlavaty, K. A., Ricci, C. F., Leonard, J. N., et al. (2016b). Transforming Growth Factor-Beta 1 Delivery from Microporous Scaffolds Decreases Inflammation post-implant and Enhances Function of Transplanted Islets. Biomaterials 80, 11-19. doi:10.1016/ j.biomaterials.2015.11.065

Liu, X. Q., Fourel, L., Dalonneau, F., Sadir, R., Leal, S., Lortat-Jacob, H., et al. (2017b). Biomaterial-enabled Delivery of SDF- $1 \alpha$ at the Ventral Side of Breast Cancer Cells Reveals a Crosstalk between Cell Receptors to Promote the Invasive Phenotype. Biomaterials 127, 61-74. doi:10.1016/ j.biomaterials.2017.02.035

Liu, X., Yang, Y., Li, Y., Niu, X., Zhao, B., Wang, Y., et al. (2017a). Integration of Stem Cell-Derived Exosomes with In Situ Hydrogel Glue as a Promising Tissue Patch for Articular Cartilage Regeneration. Nanoscale 9, 4430-4438. doi:10.1039/c7nr00352h

Lohmann, N., Schirmer, L., Atallah, P., Wandel, E., Ferrer, R. A., Werner, C., et al. (2017). Glycosaminoglycan-based Hydrogels Capture Inflammatory Chemokines and rescue Defective Wound Healing in Mice. Sci. Transl Med. 9, eaai9044. doi:10.1126/scitranslmed.aai9044

Lou, J., Stowers, R., Nam, S., Xia, Y., and Chaudhuri, O. (2018). Stress Relaxing Hyaluronic Acid-Collagen Hydrogels Promote Cell Spreading, Fiber Remodeling, and Focal Adhesion Formation in 3D Cell Culture. Biomaterials 154, 213-222. doi:10.1016/j.biomaterials.2017.11.004

Marozas, I. A., Anseth, K. S., and Cooper-White, J. J. (2019). Adaptable Boronate Ester Hydrogels with Tunable Viscoelastic Spectra to Probe Timescale Dependent Mechanotransduction. Biomaterials 223, 119430. doi:10.1016/ j.biomaterials.2019.119430

Marquardt, L. M., Doulames, V. M., Wang, A. T., Dubbin, K., Suhar, R. A., Kratochvil, M. J., et al. (2020). Designer, Injectable Gels to Prevent Transplanted Schwann Cell Loss during Spinal Cord Injury Therapy. Sci. $A d v$. 6, eaaz1039. doi:10.1126/sciadv.aaz1039

Marquez-Curtis, L. A., and Janowska-Wieczorek, A. (2013). Enhancing the Migration Ability of Mesenchymal Stromal Cells by Targeting the SDF-1/ CXCR4 axis. Biomed. Res. Int. 2013, 561098. doi:10.1155/2013/561098

Martin, J. R., Patil, P., Yu, F., Gupta, M. K., and Duvall, C. L. (2020). Enhanced Stem Cell Retention and Antioxidative protection with Injectable, ROS-Degradable PEG Hydrogels. Biomaterials 263, 120377. doi:10.1016/ j.biomaterials.2020.120377

Martino, M. M., Tortelli, F., Mochizuki, M., Traub, S., Ben-David, D., Kuhn, G. A., et al. (2011). Engineering the Growth Factor Microenvironment with Fibronectin Domains to Promote Wound and Bone Tissue Healing. Sci. Transl Med. 3, 100ra89. doi:10.1126/scitranslmed.3002614

Martino, M. M., Briquez, P. S., Güç, E., Tortelli, F., Kilarski, W. W., Metzger, S., et al. (2014). Growth Factors Engineered for Super-affinity to the Extracellular Matrix Enhance Tissue Healing. Science 343, 885-888. doi:10.1126/ science. 1247663

Mckinnon, D. D., Domaille, D. W., Brown, T. E., Kyburz, K. A., Kiyotake, E., Cha, J. N., et al. (2014a). Measuring Cellular Forces Using Bis-Aliphatic Hydrazone Crosslinked Stress-Relaxing Hydrogels. Soft matter 10, 9230-9236. doi:10.1039/ c4sm01365d

Mckinnon, D. D., Domaille, D. W., Cha, J. N., and Anseth, K. S. (2014b). Biophysically Defined and Cytocompatible Covalently Adaptable Networks as Viscoelastic 3D Cell Culture Systems. Adv. Mater. 26, 865-872. doi:10.1002/ adma. 201303680

Mizuta, R., Sasaki, Y., Kawasaki, R., Katagiri, K., Sawada, S.-i., Mukai, S.-a., et al. (2019). Magnetically Navigated Intracellular Delivery of Extracellular Vesicles 
Using Amphiphilic Nanogels. Bioconjug. Chem. 30, 2150-2155. doi:10.1021/ acs.bioconjchem. 9 b00369

Nam, S., Hu, K. H., Butte, M. J., and Chaudhuri, O. (2016a). Strain-enhanced Stress Relaxation Impacts Nonlinear Elasticity in Collagen Gels. Proc. Natl. Acad. Sci. USA 113, 5492-5497. doi:10.1073/pnas.1523906113

Nam, S., Lee, J., Brownfield, D. G., and Chaudhuri, O. (2016b). Viscoplasticity Enables Mechanical Remodeling of Matrix by Cells. Biophysical J. 111, 2296-2308. doi:10.1016/j.bpj.2016.10.002

Nisbet, D. R., Wang, T. Y., Bruggeman, K. F., Niclis, J. C., Somaa, F. A., Penna, V., et al. (2018). Shear Containment of BDNF within Molecular Hydrogels Promotes Human Stem Cell Engraftment and Postinfarction Remodeling in Stroke. Adv. Biosys. 2, 1800113. doi:10.1002/adbi.201800113

Niu, H., Li, C., Guan, Y., Dang, Y., Li, X., Fan, Z., et al. (2020). High Oxygen Preservation Hydrogels to Augment Cell Survival under Hypoxic Condition. Acta Biomater. 105, 56-67. doi:10.1016/j.actbio.2020.01.017

Noh, S.-S., Bhang, S. H., La, W.-G., Lee, S., Shin, J.-Y., Ma, Y.-J., et al. (2015). A Dual Delivery of Substance $\mathrm{P}$ and Bone Morphogenetic Protein-2 for Mesenchymal Stem Cell Recruitment and Bone Regeneration. Tissue Eng. A 21, 1275-1287. doi:10.1089/ten.tea.2014.0182

Paganini, C., Capasso Palmiero, U., Pocsfalvi, G., Touzet, N., Bongiovanni, A., and Arosio, P. (2019). Scalable Production and Isolation of Extracellular Vesicles: Available Sources and Lessons from Current Industrial Bioprocesses. Biotechnol. J. 14, e1800528. doi:10.1002/biot.201800528

Parisi-Amon, A., Mulyasasmita, W., Chung, C., and Heilshorn, S. C. (2013). Protein-engineered Injectable Hydrogel to Improve Retention of Transplanted Adipose-Derived Stem Cells. Adv. Healthc. Mater. 2, 428-432. doi:10.1002/adhm.201200293

Park, D. J., Yun, W. S., Kim, W. C., Park, J.-E., Lee, S. H., Ha, S., et al. (2020a). Improvement of Stem Cell-Derived Exosome Release Efficiency by SurfaceModified Nanoparticles. J. Nanobiotechnol 18, 178. doi:10.1186/s12951-02000739-7

Park, J. W., Han, S. B., Hah, J., Lee, G., Kim, J. K., Kim, S. H., et al. (2020b). Biological Aging Modulates Cell Migration via Lamin A/C-Dependent Nuclear Motion. Micromachines (Basel) 11. doi:10.3390/mi11090801

Park, J., Andrade, B., Seo, Y., Kim, M.-J., Zimmerman, S. C., and Kong, H. (2018). Engineering the Surface of Therapeutic "Living" Cells. Chem. Rev. 118, 1664-1690. doi:10.1021/acs.chemrev.7b00157

Park, T. Y., Oh, J.-M., Cho, J. S., Sim, S. B., Lee, J., and Cha, H. J. (2020c). Stem CellLoaded Adhesive Immiscible Liquid for Regeneration of Myocardial Infarction. J. Controlled Release 321, 602-615. doi:10.1016/j.jconrel.2020.02.047

Parmar, M., Grealish, S., and Henchcliffe, C. (2020). The Future of Stem Cell Therapies for Parkinson Disease. Nat. Rev. Neurosci. 21, 103-115. doi:10.1038/ s41583-019-0257-7

Pavlovic, M. (2015). "What Are Biomaterials," in Bioengineering (Cham: Springer International Publishing), 229-244. doi:10.1007/978-3-319-10798-1_18

Pelham, R. J., Jr., and Wang, Y.-l. (1997). Cell Locomotion and Focal Adhesions Are Regulated by Substrate Flexibility. Proc. Natl. Acad. Sci. 94, 13661-13665. doi:10.1073/pnas.94.25.13661

Petit, I., Jin, D., and Rafii, S. (2007). The SDF-1-CXCR4 Signaling Pathway: a Molecular Hub Modulating Neo-Angiogenesis. Trends Immunol. 28, 299-307. doi:10.1016/j.it.2007.05.007

Purcell, B. P., Lobb, D., Charati, M. B., Dorsey, S. M., Wade, R. J., Zellars, K. N., et al. (2014). Injectable and Bioresponsive Hydrogels for On-Demand Matrix Metalloproteinase Inhibition. Nat. Mater 13, 653-661. doi:10.1038/nmat3922

Qin, E. C., Ahmed, S. T., Sehgal, P., Vu, V. H., Kong, H., and Leckband, D. E. (2020). Comparative Effects of N-Cadherin Protein and Peptide Fragments on Mesenchymal Stem Cell Mechanotransduction and Paracrine Function. Biomaterials 239, 119846. doi:10.1016/j.biomaterials.2020.119846

Rafique, M., Wei, T., Sun, Q., Midgley, A. C., Huang, Z., Wang, T., et al. (2021). The Effect of Hypoxia-Mimicking Responses on Improving the Regeneration of Artificial Vascular Grafts. Biomaterials 271, 120746. doi:10.1016/ j.biomaterials.2021.120746

Richardson, B. M., Wilcox, D. G., Randolph, M. A., and Anseth, K. S. (2019). Hydrazone Covalent Adaptable Networks Modulate Extracellular Matrix Deposition for Cartilage Tissue Engineering. Acta Biomater. 83, 71-82. doi:10.1016/j.actbio.2018.11.014

Rinker, T. E., Philbrick, B. D., Hettiaratchi, M. H., Smalley, D. M., Mcdevitt, T. C., and Temenoff, J. S. (2018). Microparticle-mediated Sequestration of Cell-
Secreted Proteins to Modulate Chondrocytic Differentiation. Acta Biomater. 68, 125-136. doi:10.1016/j.actbio.2017.12.038

Rosales, A. M., and Anseth, K. S. (2016). The Design of Reversible Hydrogels to Capture Extracellular Matrix Dynamics. Nat. Rev. Mater. 1, 1-15. doi:10.1038/ natrevmats.2015.12

Shafiq, M., Zhang, Q., Zhi, D., Wang, K., Kong, D., Kim, D. H., et al. (2018). In Situ Blood Vessel Regeneration Using SP (Substance P) and SDF (Stromal CellDerived Factor)-1a Peptide Eluting Vascular Grafts. Arterioscler Thromb. Vasc. Biol. 38, e117-e134. doi:10.1161/ATVBAHA.118.310934

Shafiq, M., Jung, Y., and Kim, S. H. (2016). Insight on Stem Cell Preconditioning and Instructive Biomaterials to Enhance Cell Adhesion, Retention, and Engraftment for Tissue Repair. Biomaterials 90, 85-115. doi:10.1016/ j.biomaterials.2016.03.020

Shafiq, M., Kong, D., and Kim, S. H. (2017). SDF-1 $\alpha$ Peptide Tethered Polyester Facilitates Tissue Repair by Endogenous Cell Mobilization and Recruitment. J. Biomed. Mater. Res. 105, 2670-2684. doi:10.1002/jbm.a.36130

Shevach, M., Zax, R., Abrahamov, A., Fleischer, S., Shapira, A., and Dvir, T. (2015). Omentum ECM-Based Hydrogel as a Platform for Cardiac Cell Delivery. Biomed. Mater. 10, 034106. doi:10.1088/1748-6041/10/3/034106

Sneider, A., Hah, J., Wirtz, D., and Kim, D.-H. (2019). Recapitulation of Molecular Regulators of Nuclear Motion during Cell Migration. Cell Adhes. Migration 13, 50-62. doi:10.1080/19336918.2018.1506654

Somaa, F. A., Wang, T.-Y., Niclis, J. C., Bruggeman, K. F., Kauhausen, J. A., Guo, H., et al. (2017). Peptide-Based Scaffolds Support Human Cortical Progenitor Graft Integration to Reduce Atrophy and Promote Functional Repair in a Model of Stroke. Cel Rep. 20, 1964-1977. doi:10.1016/j.celrep.2017.07.069

Swanson, W. B., Gong, T., Zhang, Z., Eberle, M., Niemann, D., Dong, R., et al. (2020). Controlled Release of Odontogenic Exosomes from a Biodegradable Vehicle Mediates Dentinogenesis as a Novel Biomimetic Pulp Capping Therapy. J. Controlled Release 324, 679-694. doi:10.1016/j.jconrel.2020.06.006

Tang, J., Wang, J., Huang, K., Ye, Y., Su, T., Qiao, L., et al. (2018). Cardiac CellIntegrated Microneedle Patch for Treating Myocardial Infarction. Sci. Adv. 4, eaat9365. doi:10.1126/sciadv.aat9365

Teo, J. Y., Ko, E., Leong, J., Hong, J., Jeon, J. S., Yang, Y. Y., et al. (2020). Surface Tethering of Stromal Cell-Derived Factor-1a Carriers to Stem Cells Enhances Cell Homing to Ischemic Muscle. Nanomedicine: Nanotechnology, Biol. Med. 28, 102215. doi:10.1016/j.nano.2020.102215

Théry, C., Witwer, K. W., Aikawa, E., Alcaraz, M. J., Anderson, J. D., Andriantsitohaina, R., et al. (2018). Minimal Information for Studies of Extracellular Vesicles 2018 (MISEV2018): a Position Statement of the International Society for Extracellular Vesicles and Update of the MISEV2014 Guidelines. J. Extracellular Vesicles 7, 1535750. doi:10.1080/ 20013078.2018.1535750

Trounson, A., and Mcdonald, C. (2015). Stem Cell Therapies in Clinical Trials: Progress and Challenges. Cell Stem Cell 17, 11-22. doi:10.1016/j.stem.2015.06.007

Ullah, I., Subbarao, R. B., and Rho, G. J. (2015). Human Mesenchymal Stem Cells Current Trends and Future Prospective. Biosci. Rep. 35, e00191. doi:10.1042/ BSR20150025

Vandergriff, A., Huang, K., Shen, D., Hu, S., Hensley, M. T., Caranasos, T. G., et al. (2018). Targeting Regenerative Exosomes to Myocardial Infarction Using Cardiac Homing Peptide. Theranostics 8, 1869-1878. doi:10.7150/thno.20524

Wang, H., Shang, Y., Chen, X., Wang, Z., Zhu, D., Liu, Y., et al. (2020). Delivery of MSCs with a Hybrid $\beta$-Sheet Peptide Hydrogel Consisting IGF-1C Domain and D-form Peptide for Acute Kidney Injury Therapy. Int. J. Nanomedicine 15, 4311-4324. doi:10.2147/ijn.s254635

Wang, M., Altinoglu, S., Takeda, Y. S., and Xu, Q. (2015). Integrating Protein Engineering and Bioorthogonal Click Conjugation for Extracellular Vesicle Modulation and Intracellular Delivery. PLoS One 10, e0141860. doi:10.1371/ journal.pone.0141860

Wang, S., Wong Po Foo, C., Warrier, A., Poo, M.-m., Heilshorn, S. C., and Zhang, X. (2009). Gradient Lithography of Engineered Proteins to Fabricate 2D and 3D Cell Culture Microenvironments. Biomed. Microdevices 11, 1127-1134. doi:10.1007/s10544-009-9329-1

Wang, T.-Y., Bruggeman, K. F., Kauhausen, J. A., Rodriguez, A. L., Nisbet, D. R., and Parish, C. L. (2016). Functionalized Composite Scaffolds Improve the Engraftment of Transplanted Dopaminergic Progenitors in a Mouse Model of Parkinson's Disease. Biomaterials 74, 89-98. doi:10.1016/ j.biomaterials.2015.09.039 
Wozniak, M. A., and Chen, C. S. (2009). Mechanotransduction in Development: a Growing Role for Contractility. Nat. Rev. Mol. Cel Biol 10, 34-43. doi:10.1038/ nrm 2592

Xiang, X., Liu, H., Wang, L., Zhu, B., Ma, L., Du, F., et al. (2020). Ultrasound Combined with SDF-1a Chemotactic Microbubbles Promotes Stem Cell Homing in an Osteoarthritis Model. J. Cel Mol Med 24, 10816-10829. doi:10.1111/jcmm.15706

Yang, B., Wei, K., Loebel, C., Zhang, K., Feng, Q., Li, R., et al. (2021). Enhanced Mechanosensing of Cells in Synthetic 3D Matrix with Controlled Biophysical Dynamics. Nat. Commun. 12, 3514. doi:10.1038/s41467-02123120-0

Yu, W., Su, X., Li, M., Wan, W., Li, A., Zhou, H., et al. (2021). Three-dimensional Mechanical Microenvironment Enhanced Osteogenic Activity of Mesenchymal Stem Cells-Derived Exosomes. Chem. Eng. J. 417, 128040. doi:10.1016/ j.cej.2020.128040

Zhang, K., Zhao, X., Chen, X., Wei, Y., Du, W., Wang, Y., et al. (2018a). Enhanced Therapeutic Effects of Mesenchymal Stem Cell-Derived Exosomes with an Injectable Hydrogel for Hindlimb Ischemia Treatment. ACS Appl. Mater. Inter. 10, 30081-30091. doi:10.1021/acsami.8b08449

Zhang, Q., Nguyen, P. D., Shi, S., Burrell, J. C., Cullen, D. K., and Le, A. D. (2018b). 3D Bio-Printed Scaffold-free Nerve Constructs with Human Gingiva-Derived Mesenchymal Stem Cells Promote Rat Facial Nerve Regeneration. Sci. Rep. 8, 6634. doi:10.1038/s41598-018-24888-w

Zhang, Y., Cai, Z., Shen, Y., Lu, Q., Gao, W., Zhong, X., et al. (2021). Hydrogel-load Exosomes Derived from Dendritic Cells Improve Cardiac Function via Treg Cells and the Polarization of Macrophages Following Myocardial Infarction. J. Nanobiotechnol 19, 271. doi:10.1186/s12951-021-01016-x

Zhang, Y., Qi, Y., Ulrich, S., Barboiu, M., and Ramström, O. (2020). Dynamic Covalent Polymers for Biomedical Applications. Mater. Chem. Front. 4, 489-506. doi:10.1039/c9qm00598f
Zhao, X., Cui, K., and Li, Z. (2019). The Role of Biomaterials in Stem Cell-Based Regenerative Medicine. Future Med. Chem. 11, 1777-1790. doi:10.4155/fmc2018-0347

Zhou, Y., Liu, S., Zhao, M., Wang, C., Li, L., Yuan, Y., et al. (2019). Injectable Extracellular Vesicle-Released Self-Assembling Peptide Nanofiber Hydrogel as an Enhanced Cell-free Therapy for Tissue Regeneration. J. Controlled Release 316, 93-104. doi:10.1016/j.jconrel.2019.11.003

Zhu, W., Sun, L., Zhao, P., Liu, Y., Zhang, J., Zhang, Y., et al. (2021). Macrophage Migration Inhibitory Factor Facilitates the Therapeutic Efficacy of Mesenchymal Stem Cells Derived Exosomes in Acute Myocardial Infarction through Upregulating miR-133a-3p. J. Nanobiotechnol 19, 61. doi:10.1186/ s12951-021-00808-5

Conflict of Interest: The authors declare that the research was conducted in the absence of any commercial or financial relationships that could be construed as a potential conflict of interest.

Publisher's Note: All claims expressed in this article are solely those of the authors and do not necessarily represent those of their affiliated organizations, or those of the publisher, the editors and the reviewers. Any product that may be evaluated in this article, or claim that may be made by its manufacturer, is not guaranteed or endorsed by the publisher.

Copyright (c) 2021 Shafiq, Ali, Han and Kim. This is an open-access article distributed under the terms of the Creative Commons Attribution License (CC $B Y)$. The use, distribution or reproduction in other forums is permitted, provided the original author(s) and the copyright owner(s) are credited and that the original publication in this journal is cited, in accordance with accepted academic practice. No use, distribution or reproduction is permitted which does not comply with these terms. 